\title{
Alkynyltrifluoroborates as Versatile Tools in Organic Synthesis: A New Route to Spiroketals.
}

\author{
Jan Doubský, Ludvík Streinz, David Šaman, Jiří Zedník, and Bohumír Koutek
}

\section{General}

All reactions were carried out under an Ar atmosphere using oven dried glassware and standard syringe and septa techniques. All work-up, wash, and chromatographic solvents were distilled prior to use. Diethyl ether and THF were distilled from $\mathrm{Na}$ /benzophenone under $\mathrm{Ar}$.

Benzyl protected alkynols 1 were prepared according to the standard procedure from corresponding alcohols and benzyl bromide in the presence of $\mathrm{NaH}$ in $\mathrm{THF}^{1}$. Starting alkynols, lactones and $\mathrm{BF}_{3} \cdot \mathrm{Et}_{2} \mathrm{O}$ are commercially available and were used without further purification.

Gas chromatography was used to monitor the progress of reactions (Hewlett-Packard HP 6890 series equipped with flame ionization detector; capillary column with $5 \%$ phenyl-methyl-siloxane as stationary phase (30 $\mathrm{m} \times 0.32 \mathrm{~mm}$ I.D., $0.25 \mu \mathrm{m}$ film thickness) and helium as a carrier gas). Chiral gas chromatography analyses were performed using fused silica capillary column with $20 \%$ of permethylated $\beta$-cyclodextrin in poly(35\% diphenyl/ $65 \%$ dimethylsiloxane) as the stationary phase (30 $\mathrm{m} \times 0.25 \mathrm{~mm}$ I.D., $0.25 \mu \mathrm{m}$ film thickness).

Flash chromatographic purifications were performed using Merck Kieselgel 60 (230-400 mesh).

${ }^{1} \mathrm{H}-\mathrm{NMR}$ and ${ }^{13} \mathrm{C}-\mathrm{NMR}$ data were recorded on Varian Unity-200, Varian Unity Inova-400, Bruker Avance 400, and Bruker Avance 500 in $\mathrm{CDCl}_{3}$ and all chemical shifts are given in parts per million (ppm) downfield from tetramethylsilane as internal standard $\left({ }^{1} \mathrm{H}\right)$ or relative to $\mathrm{CDCl}_{3}\left({ }^{13} \mathrm{C}\right)$. Peak multiplicates in ${ }^{1} \mathrm{H}$-NMR spectra are abbreviated as s (singlet), $d$ (doublet), $t$ (triplet), $m$ (multiplet), and bs (broad singlet).

Interaction constants $\mathrm{J}$ are given in $\mathrm{Hz}$ (rounded to one decimal place).

Gas chromatography-mass spectrometry was performed on a Carlo Erba GC 8000 series gas chromatograph (capillary column with $5 \%$ phenyl-methyl-siloxane as stationary phase, $30 \mathrm{~m} \times 0.25 \mathrm{~mm}$ I.D., $0.25 \mu \mathrm{m}$ film thickness) and Fisons MD 800 mass detector (quadrupole mass spectrometer using 70 $\mathrm{eV}$ electron impact ionization).

Infrared spectra were recorded with a Bruker IFS 88 and a Bruker Equinox 55 Fourier-transform infrared spectrometers. Absorption maxima are expressed in wavenumbers $\left(\mathrm{cm}^{-1}\right)$ with possible abbreviation br (broad). 


\section{Synthesis of Alkynones 3:}

To a solution of alkyne $1(8 \mathrm{mmol})$ in dry THF $(25 \mathrm{ml})$ at $-78^{\circ} \mathrm{C}, n \mathrm{BuLi}(5 \mathrm{ml}, 1.6 \mathrm{M}, 8 \mathrm{mmol})$ was slowly added, maintaining an argon atmosphere. The reaction mixture was stirred for $20 \mathrm{~min}$, allowed to warm to about $-30^{\circ} \mathrm{C}$, cooled to $-78^{\circ} \mathrm{C}$ again, and $\mathrm{BF}_{3} \cdot \mathrm{Et}_{2} \mathrm{O}(1 \mathrm{ml}, 7.9 \mathrm{mmol})$ was added dropwise. Stirring was continued for $10 \mathrm{~min}$, and then lactone $2(7.2 \mathrm{mmol})$ was added in one portion via syringe. The resulting mixture was allowed to warm to room temperature within $1 \mathrm{~h}$, a solution of saturated $\mathrm{NH}_{4} \mathrm{Cl}-$

$\mathrm{NH}_{3}$ (aq.) 2:1 (5 ml) was added, the mixture was poured into water $(50 \mathrm{ml})$, and extracted with $\mathrm{Et}_{2} \mathrm{O}(4 \times 25$ $\mathrm{ml}$ ). The combined ethereal layers were washed with brine, dried briefly with $\mathrm{MgSO}_{4}$, and evaporated to afford the crude product as a slightly yellow oil. The crude material was purified by chromatography on silica gel (hexane / 10-30\% EtOAc) to yield the alkynone 3.

\section{Synthesis of Spiroketals 4:}

Alkynone $3(5 \mathrm{mmol})$ was dissolved in $50 \mathrm{ml}$ of anhydrous EtOH or EtOAc, and $250 \mathrm{mg}$ of $10 \%$ $\mathrm{Pd} / \mathrm{C}$ was added. The mixture was stirred under $\mathrm{H}_{2}(1 \mathrm{~atm})$ at room temperature while monitoring the progress of reaction by GC. After the completion of reaction, the catalyst was filtered off, washed with the same solvent used for the reaction, and the most of the solvent was distilled off under atmospheric pressure. The residue could be then either directly distilled under reduced pressure to furnish the desired product 4, or diluted with water (in the case of $\mathrm{EtOH}$ ), extracted three times with pentane, dried over $\mathrm{Na}_{2} \mathrm{SO}_{4}$, and after evaporation of volatiles distilled under reduced pressure.

In some cases, the pre-formed diastereomeric and/or spiroketal/hemiketal mixtures were converted into the more stable $E$-isomers (4b, $\mathbf{4 d}, \mathbf{4 g}, \mathbf{4 i}$ and $\mathbf{4 k}$ ), or $E, E / E, Z$-mixtures (4e and $\mathbf{4 n}$ ) respectively, before the isolation: After the most of the solvent was distilled off, a few drops of $2 \mathrm{~N} \mathrm{HCl}$ was added, and the mixture was stirred for approx. $5 \mathrm{~min}$, diluted with pentane, extracted once with satd. aq. $\mathrm{NaHCO}_{3}$, and the organic phase was dried over $\mathrm{Na}_{2} \mathrm{SO}_{4}$. Further work-up followed the standard procedure described above.

The yields reported below for each of the spiroketals 4 represent isolated yields (GC purity in parenthesis). 


\section{Selected analytical data:}

Detailed analytical data of new $\alpha$-alkynones $\mathbf{3 k - n}$ are summarized below, while those of $\mathbf{3 a - j}$ were reported previously. ${ }^{2}$ All spiroketals 4 (including individual isomers) were identified by GC-MS and their spectra were in accordance with those reported in literature. ${ }^{3-16}$ Examples of selected MS, GC-IR, ${ }^{1} \mathrm{H}-\mathrm{NMR}$, and ${ }^{13} \mathrm{C}-\mathrm{NMR}$ spectra are enclosed.

\section{3k 2-Benzyloxy-11-hydroxyundec-4-yn-6-one:}

${ }^{1} \mathrm{H}$ NMR $\left(400 \mathrm{MHz}, \mathrm{CDCl}_{3}\right): \delta=1.32(\mathrm{~d}, 3 \mathrm{H}, J=6.1 \mathrm{~Hz}), 1.39(\mathrm{~m}, 2 \mathrm{H}), 1.56(\mathrm{~m}, 2 \mathrm{H}), 1.69(\mathrm{~m}, 2 \mathrm{H}), 2.55$ (dd, $1 \mathrm{H}, J=17.2$ and $6.6 \mathrm{~Hz}$ ), $2.56(\mathrm{t}, 2 \mathrm{H}, J=7.3 \mathrm{~Hz}), 2.66(\mathrm{dd}, 1 \mathrm{H}, J=17.2$ and $5.4 \mathrm{~Hz}), 3.62(\mathrm{t}, 2 \mathrm{H}, J=$ $6.6 \mathrm{~Hz}$ ), 3.76 (ddq, $1 \mathrm{H}, J=6.6,6.1$ and $5.4 \mathrm{~Hz}), 4.57(\mathrm{~m}, 2 \mathrm{H}), 7.25-7.37(\mathrm{~m}, 5 \mathrm{H}) .{ }^{13} \mathrm{C} \mathrm{NMR}(100 \mathrm{MHz}$, $\left.\mathrm{CDCl}_{3}\right): \delta=19.73,23.69,25.07,26.59,32.31,45.35,62.55,70.79,72.60,82.05,90.73,127.59(2 \mathrm{C})$, 127.69, $128.41(2 \mathrm{C}), 138.10,187.99 . \mathrm{IR}\left(\mathrm{CCl}_{4}\right): 3397(\mathrm{br}, \mathrm{OH}), 2215(\mathrm{C} \equiv \mathrm{C}), 1675(\mathrm{C}=\mathrm{O}) \mathrm{cm}^{-1}$.

\section{I 1-Benzyloxy-7-hydroxynon-2-yn-4-one:}

${ }^{1} \mathrm{H}$ NMR $\left(400 \mathrm{MHz}, \mathrm{CDCl}_{3}\right): \delta=0.95(\mathrm{t}, 3 \mathrm{H}, J=7.5 \mathrm{~Hz}), 1.49(\mathrm{~m}, 2 \mathrm{H}), 1.72(\mathrm{~m}, 1 \mathrm{H}), 1.89(\mathrm{~m}, 1 \mathrm{H}), 2.76(\mathrm{~m}$, $2 \mathrm{H}), 3.54(\mathrm{~m}, 1 \mathrm{H}), 4.33(\mathrm{~s}, 2 \mathrm{H}), 4.62(\mathrm{~s}, 2 \mathrm{H}), 7.26-7.38(\mathrm{~m}, 5 \mathrm{H}) .{ }^{13} \mathrm{C} \mathrm{NMR}\left(100 \mathrm{MHz}, \mathrm{CDCl}_{3}\right): \delta=9.85$, $30.37,30.45,41.85,56.93,72.09,72.32,85.35,87.81,128.11(2 \mathrm{C}), 128.13,128.53$ (2C), 136.69, 187.48. IR $\left(\mathrm{CCl}_{4}\right): 3418(\mathrm{br}, \mathrm{OH}), 2215(\mathrm{C} \equiv \mathrm{C}), 1681(\mathrm{C}=\mathrm{O}) \mathrm{cm}^{-1}$.

\section{3m 2-Benzyloxy-8-hydroxyundec-3-yn-5-one:}

${ }^{1} \mathrm{H}$ NMR $\left(400 \mathrm{MHz}, \mathrm{CDCl}_{3}\right): \delta=0.93(\mathrm{t}, 3 \mathrm{H}, J=7.0 \mathrm{~Hz}), 1.30-1.55(\mathrm{~m}, 4 \mathrm{H}), 1.52(\mathrm{~d}, 3 \mathrm{H}, J=6.7 \mathrm{~Hz}), 1.72$ (m, 1H), $1.89(\mathrm{~m}, 1 \mathrm{H}), 2.75(\mathrm{~m}, 2 \mathrm{H}), 3.63(\mathrm{~m}, 1 \mathrm{H}), 4.36(\mathrm{q}, 1 \mathrm{H}, J=6.7 \mathrm{~Hz}), 4.51(\mathrm{~d}, 1 \mathrm{H}, J=11.6 \mathrm{~Hz}), 4.78$ $(\mathrm{d}, 1 \mathrm{H}, J=11.6 \mathrm{~Hz}), 7.25-7.37(\mathrm{~m}, 5 \mathrm{H}) .{ }^{13} \mathrm{C} N M R\left(100 \mathrm{MHz}, \mathrm{CDCl}_{3}\right): \delta=14.00,18.78,21.30,30.95,39.80$, $41.94,64.22,70.71,71.07,84.03,91.39,127.93,127.97(2 \mathrm{C}), 128.47(2 \mathrm{C}), 137.23,187.65 . \mathrm{IR}\left(\mathrm{CCl}_{4}\right)$ : $3425(\mathrm{br}, \mathrm{OH}), 2212(\mathrm{C} \equiv \mathrm{C}), 1679(\mathrm{C}=\mathrm{O}) \mathrm{cm}^{-1}$.

\section{3n 2-Benzyloxy-10-hydroxyundec-4-yn-6-one:}

${ }^{1} \mathrm{H}$ NMR $\left(400 \mathrm{MHz}, \mathrm{CDCl}_{3}\right): \delta=1.18(\mathrm{~d}, 3 \mathrm{H}, J=6.1 \mathrm{~Hz}), 1.31(\mathrm{~d}, 3 \mathrm{H}, J=6.1 \mathrm{~Hz}), 1.39-1.85(\mathrm{~m}, 4 \mathrm{H}), 2.45$ (dd, $1 \mathrm{H}, J=16.6$ and $5.1 \mathrm{~Hz}), 2.58(\mathrm{~m}, 2 \mathrm{H}), 2.66(\mathrm{dd}, 1 \mathrm{H}, J=16.6$ and $7.4 \mathrm{~Hz}), 3.73(\mathrm{ddq}, 1 \mathrm{H}, J=7.4,6.1$ and $5.1 \mathrm{~Hz}), 3.96(\mathrm{~m}, 1 \mathrm{H}), 4.57(\mathrm{~s}, 3 \mathrm{H}), 7.24-7.37(\mathrm{~m}, 5 \mathrm{H}) .{ }^{13} \mathrm{C} \mathrm{NMR}\left(100 \mathrm{MHz}, \mathrm{CDCl}_{3}\right): \delta=19.75,20.01$, 23.59, 26.62, 38.28, 44.82, 68.11, 70.79, 72.58, 82.03, 91.00, 127.50, 127.62 (2C), 128.32 (2C), 138.08, 188.11. IR $\left(\mathrm{CCl}_{4}\right): 3417(\mathrm{br}, \mathrm{OH}), 2215(\mathrm{C} \equiv \mathrm{C}), 1675(\mathrm{C}=\mathrm{O}) \mathrm{cm}^{-1}$. 
4a 1,6-Dioxaspiro[4.5]decane: ${ }^{4}$

EtOAc, $15 \mathrm{~h}$; distillation (Kugelrohr, $125^{\circ} \mathrm{C}$ air bath / 50 Torr) afforded $505 \mathrm{mg}(71 \%)$ of clear liquid (GC $\approx 100 \%$ ).

GC-MS (EI); m/z (\%):142 (10) [M+'], 141 (2), 112 (11), 111 (6), 101 (21), 100 (14), 99 (2), 98 (6), 97 (20), 87 (100), 86 (29), 85 (9), 84 (61), 83 (21), 55 (45), 41 (47).

4b 7-Methyl-1,6-dioxaspiro[4.5]decane: ${ }^{5}$

EtOAc, $14 \mathrm{~h}$, run from $50.8 \mathrm{mmol} 3 \mathbf{b}$; distillation $\left(60.5^{\circ} \mathrm{C} / 14\right.$ Torr) afforded $6.07 \mathrm{~g}$ of $(E)-4 \mathrm{~b}(76 \%)$ as clear liquid (GC 98\%).

GC-MS (El); m/z (\%):

(E)-4b: 156 (5) [M+], 155 (2), 141 (3), 126 (3), 115 (10), 114 (5), 113 (3), 112 (19), 97 (27), 87 (98), 86 (16), 85 (11), 84 (100), 69 (12), 41 (31).

(Z)-4b: MS-spectrum was nearly identical with that of $(E)-\mathbf{4 b}$.

\section{4c 2-Methyl-1,6-dioxaspiro[4.4]nonane: ${ }^{6}$}

$\mathrm{EtOH}, 17 \mathrm{~h}$; distillation (Kugelrohr, $125^{\circ} \mathrm{C}$ air bath / 50 Torr) afforded $572 \mathrm{mg}$ (80\%) of clear liquid (GC $99 \%$, mixture of two isomers 1:1). MS-spectra were closely similar for both isomers.

GC-MS (El); m/z (\%):142 (12) [M+], 141 (5), 127 (24), 112 (11), 101 (41), 100 (24), 98 (47), 97 (23), 87 (100), 85 (34), 83 (37), 69 (7), 59 (7), 56 (71), 55 (43), 41 (23).

\section{4d (5S,7S)-7-Methyl-1,6-dioxaspiro[4.5]decane: ${ }^{5,7}$}

EtOAc, $24 \mathrm{~h}$; distillation (Kugelrohr, $130^{\circ} \mathrm{C}$ air bath / 50 Torr) afforded $484 \mathrm{mg} \mathrm{(62 \% )} \mathrm{of} \mathrm{clear} \mathrm{liquid} \mathrm{(GC}$ $99 \%$, ee $>98 \%$ ). MS-spectrum was identical with that of $(E)-4 \mathbf{b}$. See Example 1.

\section{4e 2,7-Dimethyl-1,6-dioxaspiro[4.5]decane: ${ }^{8}$}

EtOH, $15 \mathrm{~h}$; distillation (Kugelrohr, $140^{\circ} \mathrm{C}$ air bath / 35 Torr) afforded $578 \mathrm{mg} \mathrm{(68 \% )} \mathrm{of} \mathrm{clear} \mathrm{liquid} \mathrm{(GC}$ $98 \%$, mixture of $E, E$ and $Z, E$-isomer 1:1). Analytical samples of $E, E-4 \mathbf{e}, Z, E-4 \mathbf{e}$, and $E, Z / Z, Z$-mixture were obtained from the crude reaction mixture by liquid chromatography $\left(\mathrm{SiO}_{2}\right.$ deactivated with $6 \% \mathrm{H}_{2} \mathrm{O}$; pentane/Et ${ }_{2} \mathrm{O}$ 99:1 - 90:10). MS-spectra were closely similar for all isomers. See Example 2. GC-MS (EI); m/z (\%): 170 (4) [M+], 155 (5), 126 (20), 115 (16), 111 (12), 101 (100), 100 (28), 98 (91), 97 (32), 85 (17), 83 (44), 69 (22), 55 (41).

\section{4f 1,7-Dioxaspiro[5.5]undecane: ${ }^{9}$}

EtOAc, $22 \mathrm{~h}$; distillation (Kugelrohr, $140^{\circ} \mathrm{C}$ air bath / 35 Torr) afforded $493 \mathrm{mg} \mathrm{(63 \% )} \mathrm{of} \mathrm{clear} \mathrm{liquid} \mathrm{(GC}$ $97 \%)$.

GC-MS (El); m/z (\%):156 (14) [M+'], 126 (3), 111 (30), 101 (88), 100 (55), 99 (9), 98 (100), 83 (55), 55 (94). 
4g 2-Methyl-1,7-dioxaspiro[5.5]undecane: ${ }^{10}$

$\mathrm{EtOH}, 28 \mathrm{~h}$; distillation (Kugelrohr, $140^{\circ} \mathrm{C}$ air bath / $35 \mathrm{Torr}$ ) afforded $553 \mathrm{mg}(65 \%)$ of $(E)-\mathbf{4 g}$ as clear liquid (GC 98\%).

GC-MS (EI); m/z (\%):

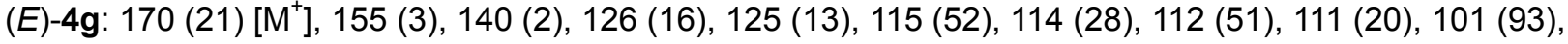
100 (37), 98 (100), 97 (39), 83 (50), 73 (16), 69 (27), 55 (62).

(Z)-4g: MS-spectrum was nearly identical with that of $(E)-\mathbf{4 g}$.

4h 2-Methyl-1,6-dioxaspiro[4.5]decane: ${ }^{5,7}$

EtOH, $26 \mathrm{~h}$; run from $12.5 \mathrm{mmol} 3 \mathrm{~h}$; distillation (Kugelrohr, $125^{\circ} \mathrm{C}$ air bath / 40 Torr) afforded $1.42 \mathrm{~g} \mathrm{(73 \% )}$ of clear liquid (GC 98\%, mixture of two isomers 1:2). MS-spectra were closely similar for both isomers. GC-MS (EI); m/z (\%): 156 (6) [M+'], 155 (2), 141 (5), 126 (2), 112 (17), 111 (18), 101 (100), 100 (44), 98 (61), 85 (13), 83 (55), 55 (48).

4i (2S,6R)-2-Methyl-1,7-dioxaspiro[5.5]undecane: ${ }^{10}$

$\mathrm{EtOH}, 26 \mathrm{~h}$; distillation (Kugelrohr, $140^{\circ} \mathrm{C}$ air bath / 35 Torr) afforded $547 \mathrm{mg}(64 \%)$ of $(2 S, 6 R)-4 \mathrm{i}$ as clear liquid (GC 99\%, ee >98\%). MS-spectrum was identical with that of $(E)-\mathbf{4 g}$. See Example 3.

4j 1,7-Dioxaspiro[5.6]dodecane: ${ }^{11}$

$\mathrm{EtOH}, 40 \mathrm{~h}$; distillation (Kugelrohr, $140^{\circ} \mathrm{C}$ air bath / 20 Torr) afforded $462 \mathrm{mg}$ (54\%) of clear liquid (GC $\approx 100 \%)$. See Example 4.

GC-MS (El); m/z (\%): 170 (7) [M+1], 125 (5), 115 (21), 112 (20), 111 (49), 101 (80), 98 (95), 97 (26), 83 (41), 73 (11), 69 (28), 55 (100).

\section{4k 2-Methyl-1,7-dioxaspiro[5.6]dodecane: ${ }^{12}$}

$\mathrm{EtOH}, 18 \mathrm{~h}$; run from $8.1 \mathrm{mmol} 3 \mathbf{k}$; distillation (Kugelrohr, $160^{\circ} \mathrm{C}$ air bath / 20 Torr) afforded $1.24 \mathrm{~g} \mathrm{(83 \% )}$ of $(E)-\mathbf{4 k}$ as clear liquid (GC $\approx 100 \%)$.

GC-MS (El); m/z (\%): 184 (11) [M+'], 169 (5),154 (7), 140 (8), 115 (100), 112 (93), 97 (49).

\section{I 2-Ethyl-1,6-dioxaspiro[4.4]nonane (Chalcogran): ${ }^{13}$}

EtOH, $24 \mathrm{~h}$; distillation (Kugelrohr, $130^{\circ} \mathrm{C}$ air bath / 35 Torr) afforded $593 \mathrm{mg}$ (76\%) of clear liquid (GC $\approx 100 \%$, mixture of two isomers $1: 1)$. MS-spectra were closely similar for both isomers.

GC-MS (El); m/z (\%): 156 (3) [M+'], 155 (3), 128 (8), 127 (100), 115 (5), 98 (25), 97 (15), 87 (32), 85 (27), 69 (10), 55 (16). 
4m 2-Methyl-7-propyl-1,6-dioxaspiro[4.4]nonane: ${ }^{3,14}$

$\mathrm{EtOH}, 37 \mathrm{~h}$; run from $2.95 \mathrm{mmol} 3 \mathrm{~m}$; distillation (Kugelrohr, $150^{\circ} \mathrm{C}$ air bath / $25 \mathrm{Torr}$ ) afforded $318 \mathrm{mg}$ $(58 \%$ ) of clear liquid ( $G C \approx 100 \%$, mixture of four isomers $3: 3: 2: 4)$. MS-spectra were closely similar for all isomers. See Example 5.

GC-MS (EI); m/z (\%): 184 (2) [M+'], 169 (4), 141 (100), 129 (12), 112 (25), 111 (26), 101 (52), 99 (7), 98 (4), 85 (68), 83 (32), 55 (38), 43 (24).

4n 2,8-Dimethyl-1,7-dioxaspiro[5.5]undecane: ${ }^{15,16}$

EtOAc, $24 \mathrm{~h}$; distillation (Kugelrohr, $160^{\circ} \mathrm{C}$ air bath / 25 Torr) afforded $549 \mathrm{mg}(60 \%)$ of clear liquid (GC $98 \%$, mixture of $E, E$ - and $E, Z$-isomer $1: 1)$.

GC-MS (El); $m / z(\%)$ :

$(E, E)-4 n: 184(12)\left[\mathrm{M}^{+}\right], 169$ (4), 140 (16), 115 (94), 114 (38), 112 (100), 97 (52), 69 (50), 55 (62), 43 (77), $41(56)$.

$(E, Z)-\mathbf{4 n}$ and $(Z, Z)-\mathbf{4 n}:$ MS-spectra were closely similar with that of $(E, E)-\mathbf{4 n}$.

\section{Literature:}

${ }^{1}$ For example see: Dupont, J.; Donato, A.J. Tetrahedron Asymm. 1998, 9, 949-954.

2 Doubský, J.; Streinz, L.; Lešetický, L.; Koutek, B. Synlett 2003, 937-942.

${ }^{3}$ For overview and examples of spiroketal fragmentations see: Francke, W.; Kitching, W. Curr. Org. Chem. 2001, 5, 233-251, and references cited therein.

${ }^{4}$ Koźluk, T.; Cottier, L.; Descotes, G. Tetrahedron 1981, 37, 1875-1880.

${ }^{5}$ Francke, W.; Hindorf, G.; Reith, W. Naturwissenschaften 1979, 66, 619-620.

${ }^{6}$ Francke, W.; Reith, W. Liebigs Ann. Chem. 1979, 1-10.

${ }^{7}$ Hintzer, K.; Weber, R.; Schurig, V. Tetrahedron Lett. 1981, 22, 55-58.

${ }^{8}$ O'Shea, M.G.; Kitching, W. Tetrahedron 1989, 45, 1177-1186.

${ }^{9}$ Hayes, P.; Maignan, C. Synthesis 1999, 783-786.

${ }^{10}$ Liu, H.; Cohen, T.; J. Org. Chem. 1995, 60, 2022-2025.

${ }^{11}$ Mudryk, B.; Shook, C.A.; Cohen, T. J. Am. Chem. Soc. 1990, 112, 6389-6391.

${ }^{12}$ Mori, K.; Katsurada, M. Liebigs Ann. Chem. 1984, 157-161.

${ }^{13}$ Mori, K.; Sasaki, M.; Tamada, S.; Suguro, T.; Masuda, S. Tetrahedron 1979, 35, 1601-1605.

${ }^{14}$ Enders, D.; Dahmen, W.; Dederichs, E.; Gatzweiler, W.; Weuster, P. Synthesis 1990, 1013-1019.

${ }^{15}$ Kitching, W.; Lewis, J.A.; Perkins, M.V.; Drew, R.; Moore, C.J., Schurig, V.; König, W.A.; Francke, W. J. Org. Chem. 1989, 54, 3893-3902.

${ }^{16}$ Perkins, M.V.; Kitching, W.; König, W.A.; Drew, R.A.I. J. Chem. Soc. Perkin Trans. I 1990, 2501-2506. 


\section{Example 1:}

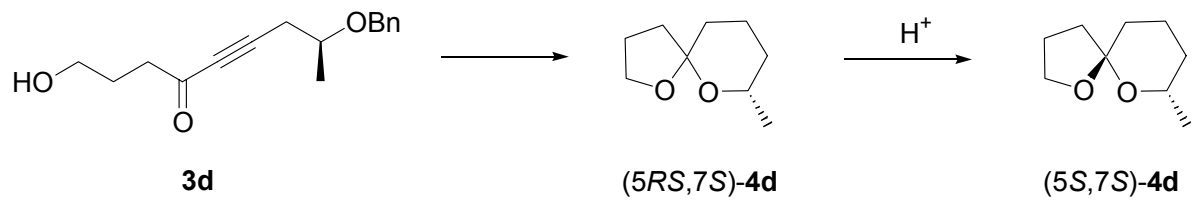

\section{GC analyses:}

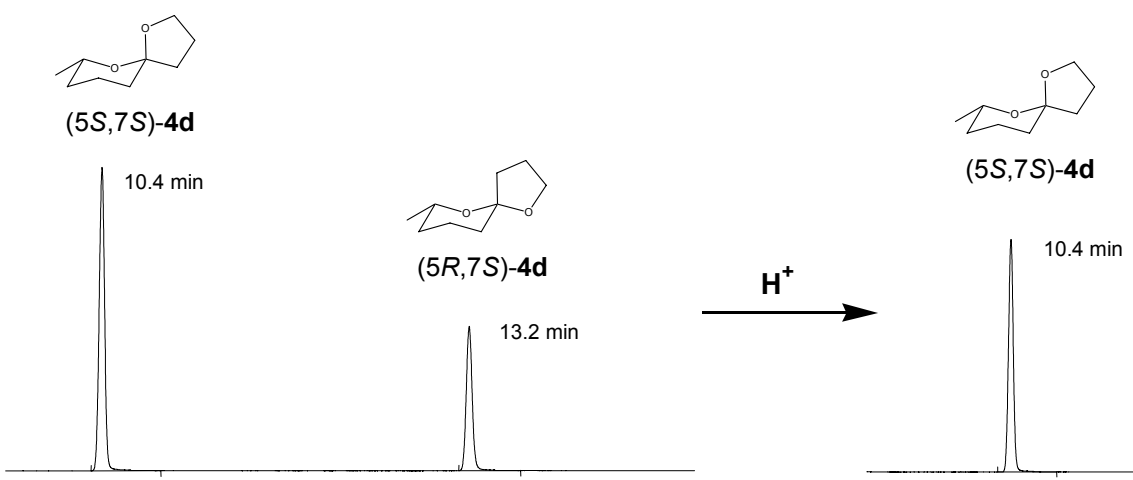

\section{GC-MS:}

MS-spectra were closely similar for both isomers.

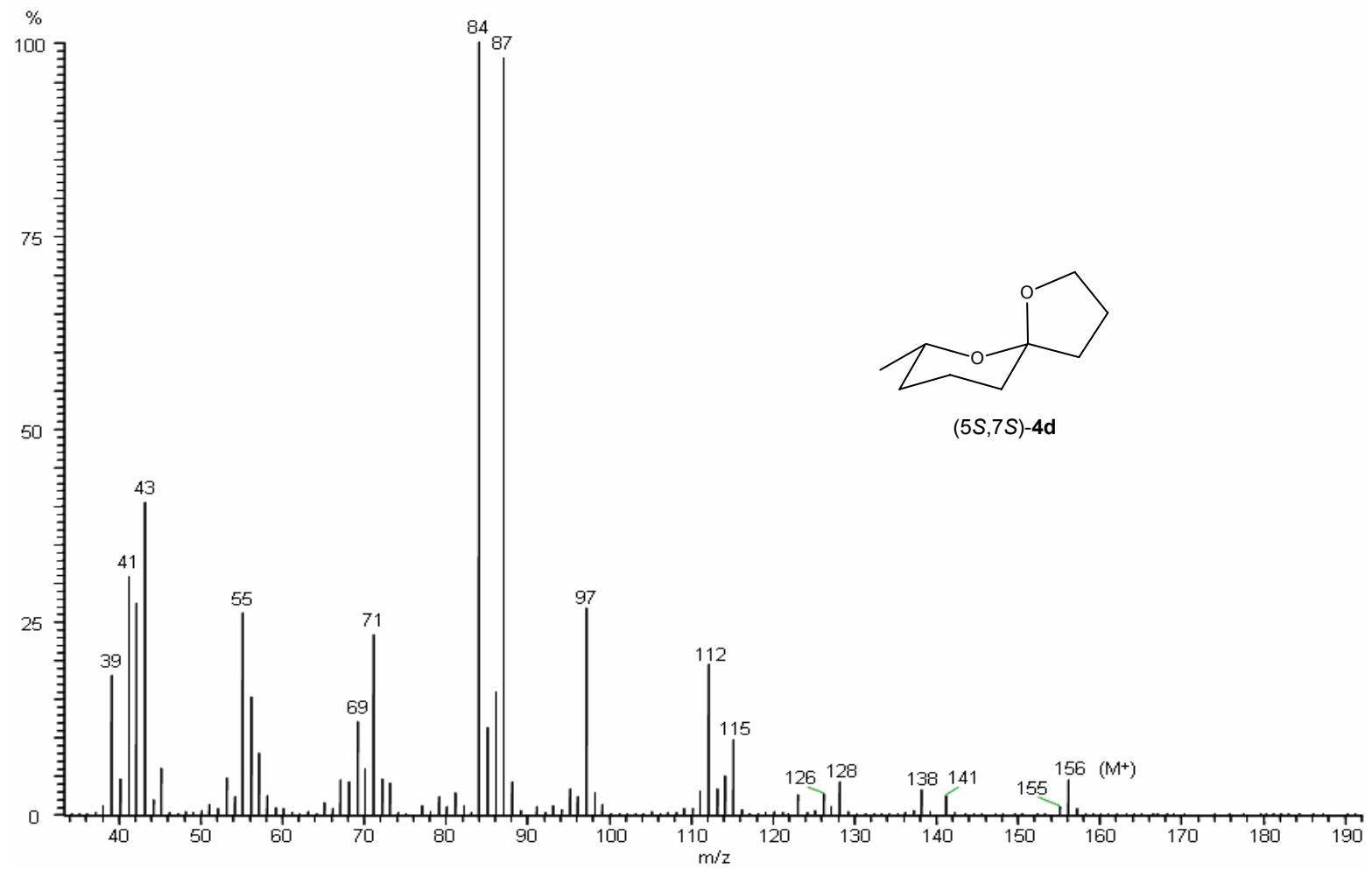


GC-IR:
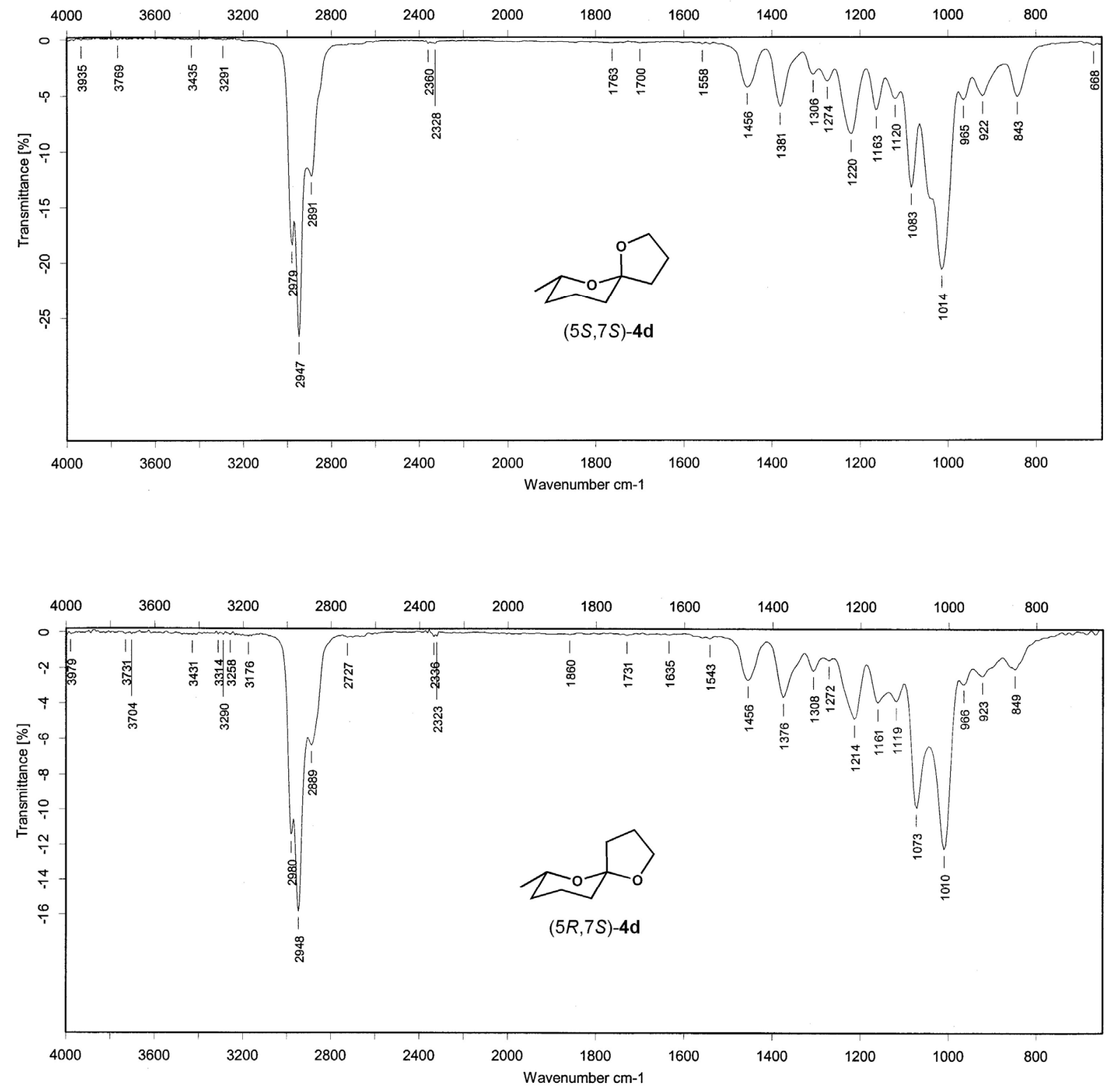


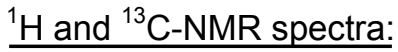
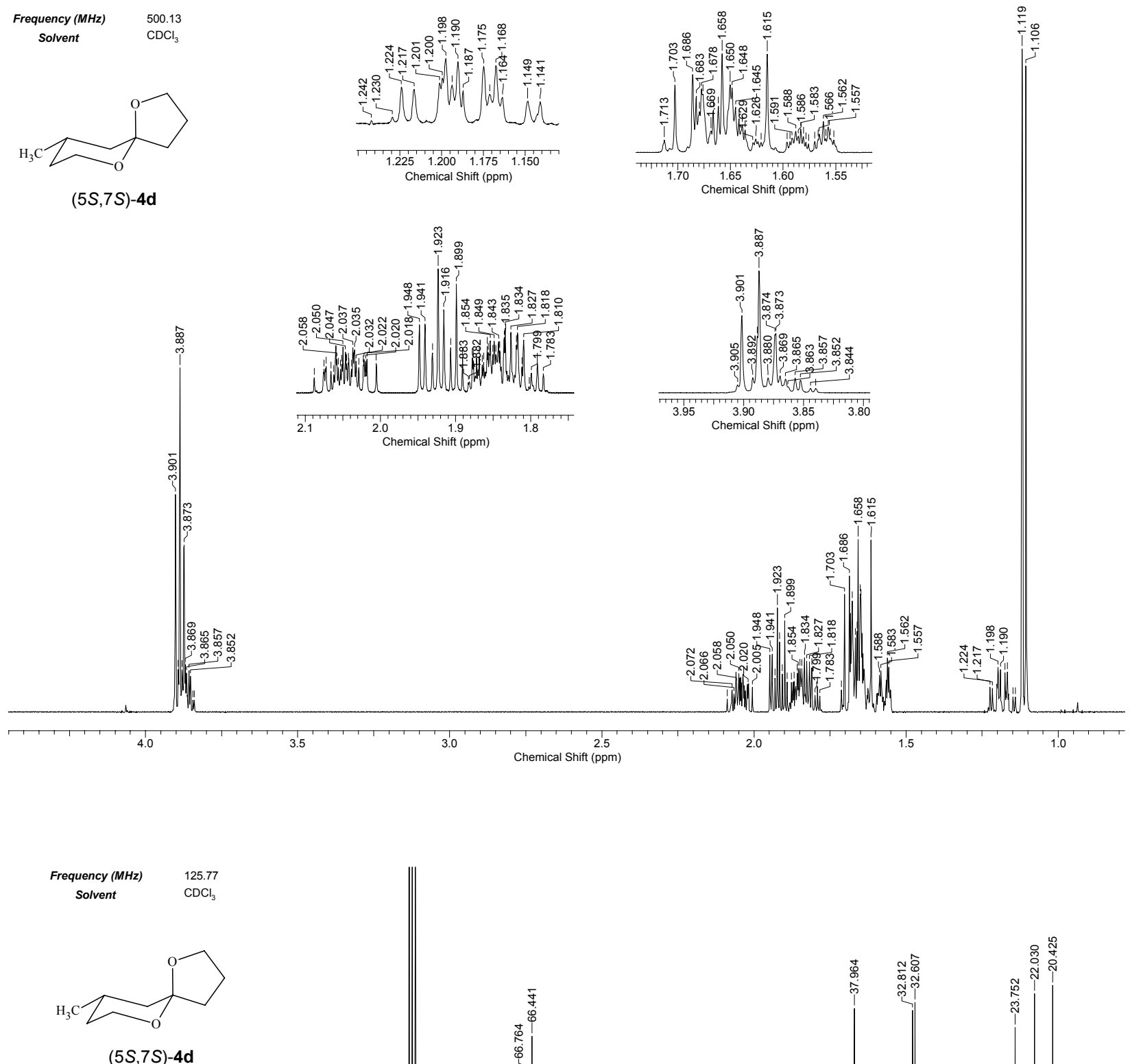

(5S,7S)-4d

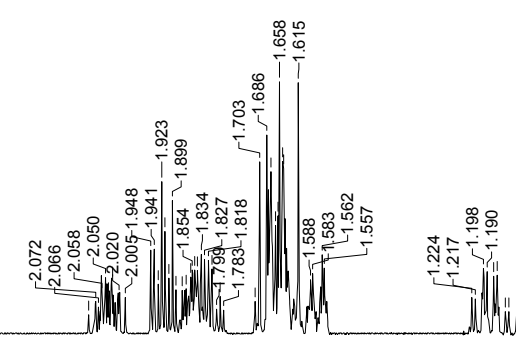




\section{Example 2:}

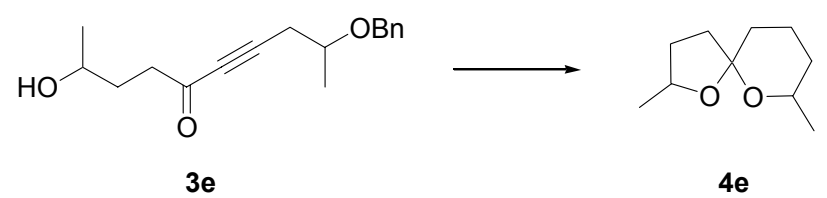

\section{$\underline{\text { GC analyses: }}$}

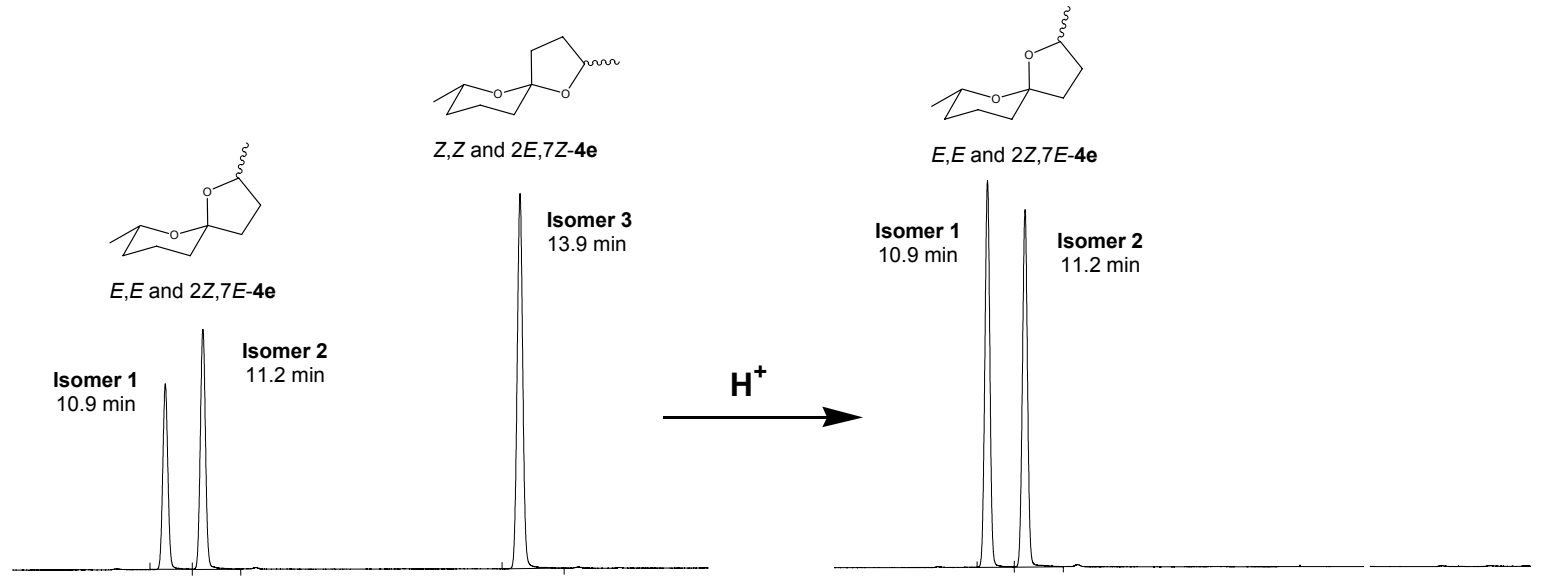

GC-MS:

MS-spectra were closely similar for all isomers.

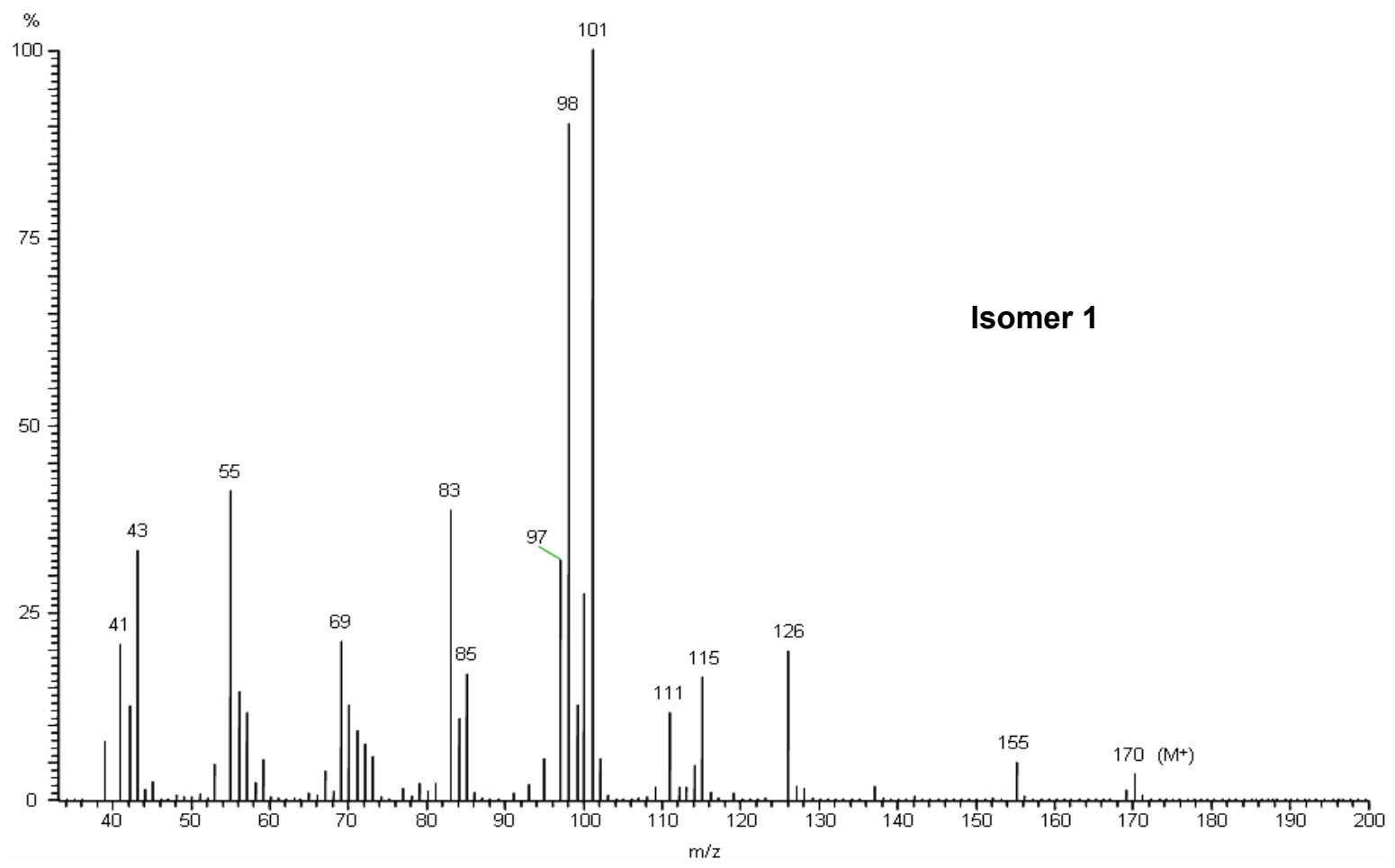


GC-IR:
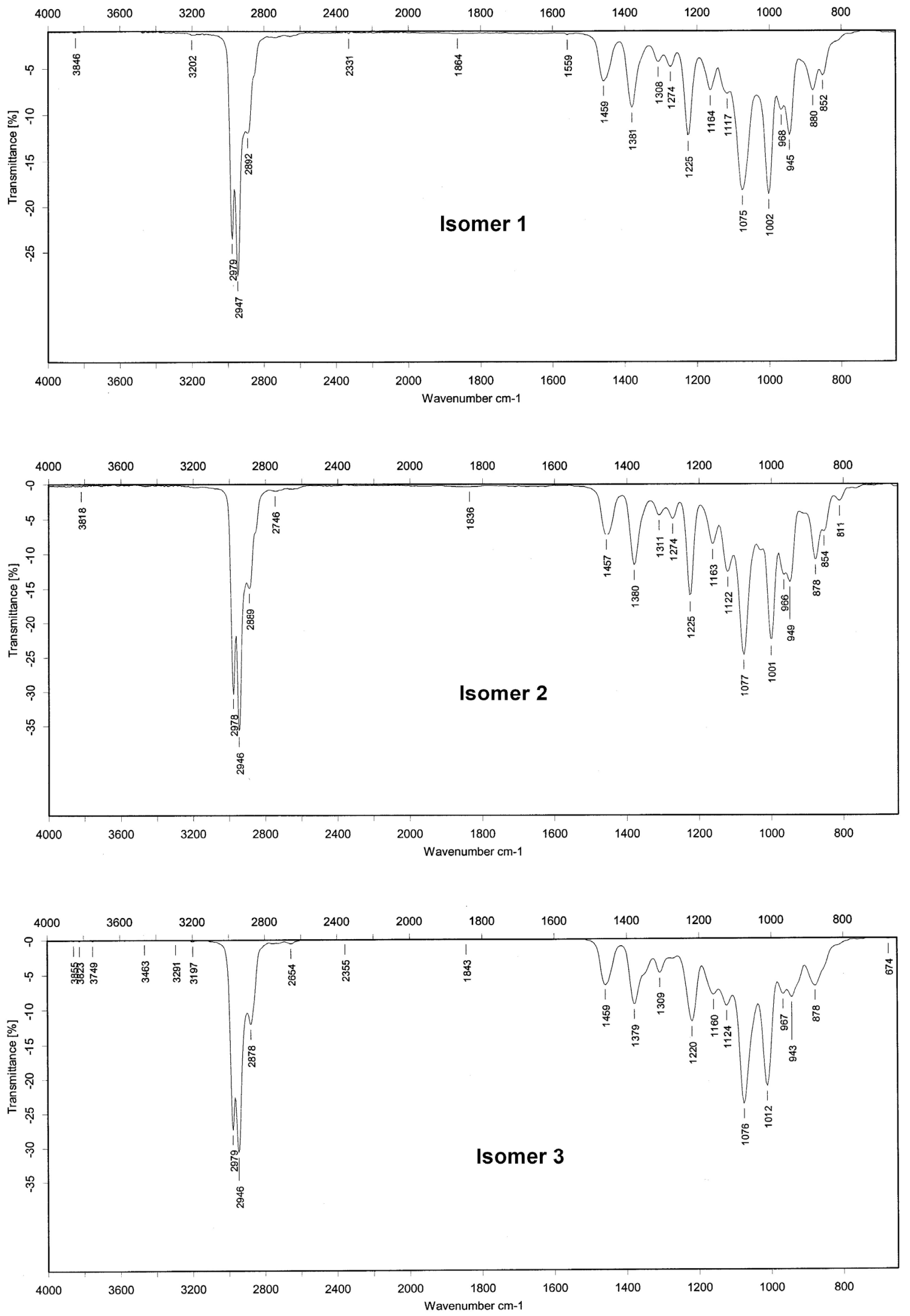


\section{${ }^{1} \mathrm{H}$ and ${ }^{13} \mathrm{C}-$ NMR spectra:}
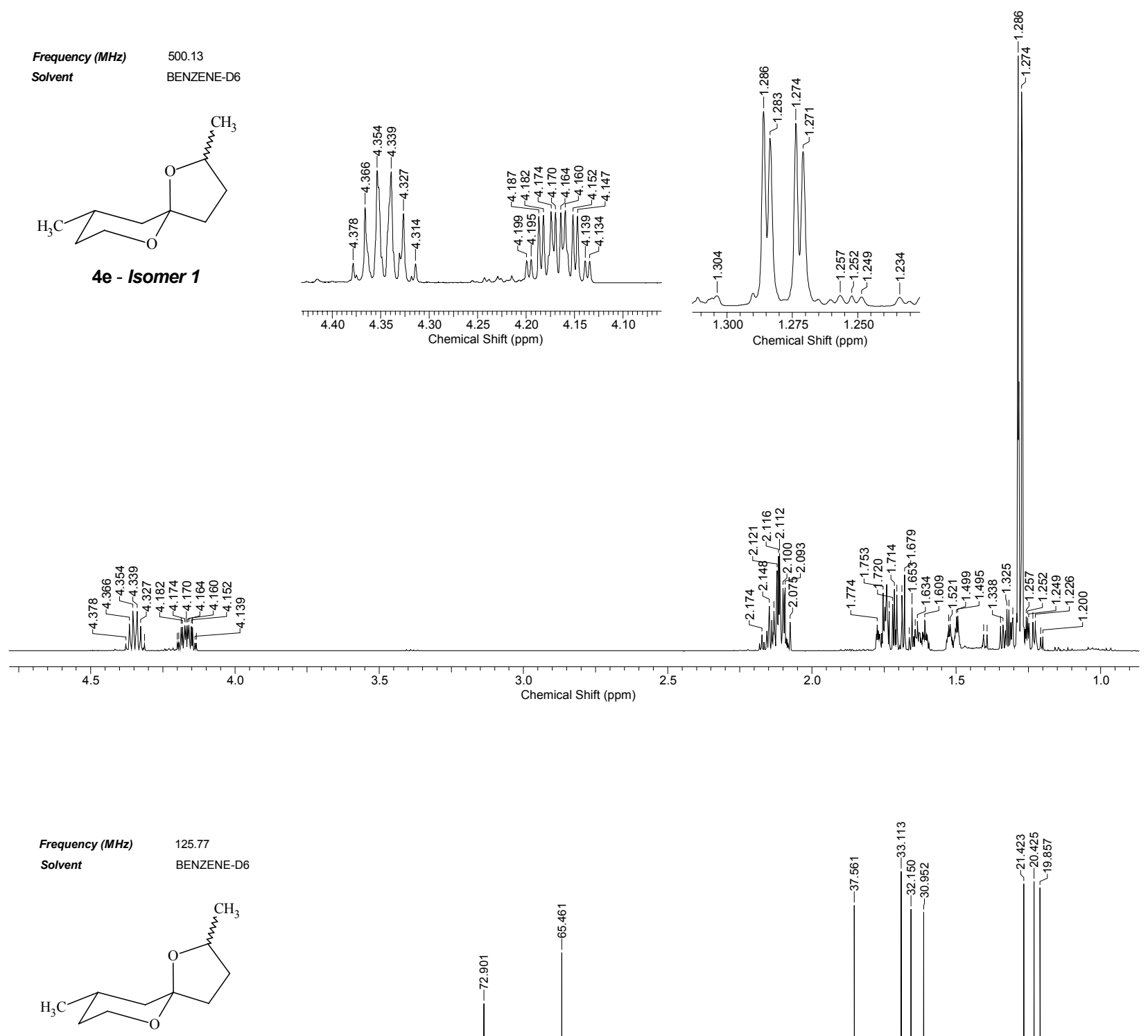

4e - Isomer 1

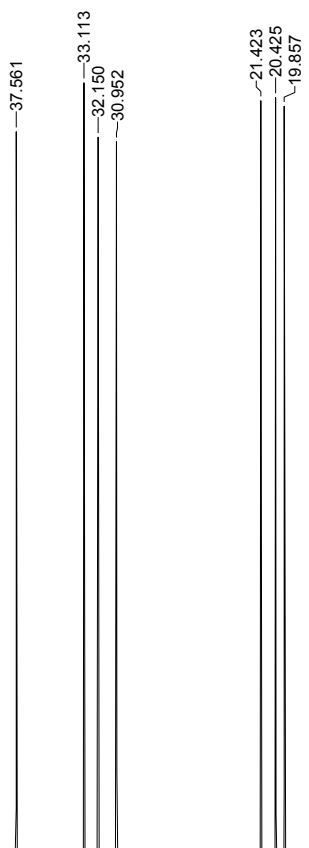

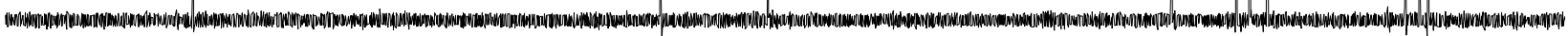

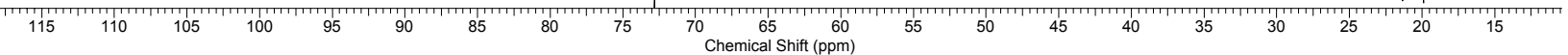



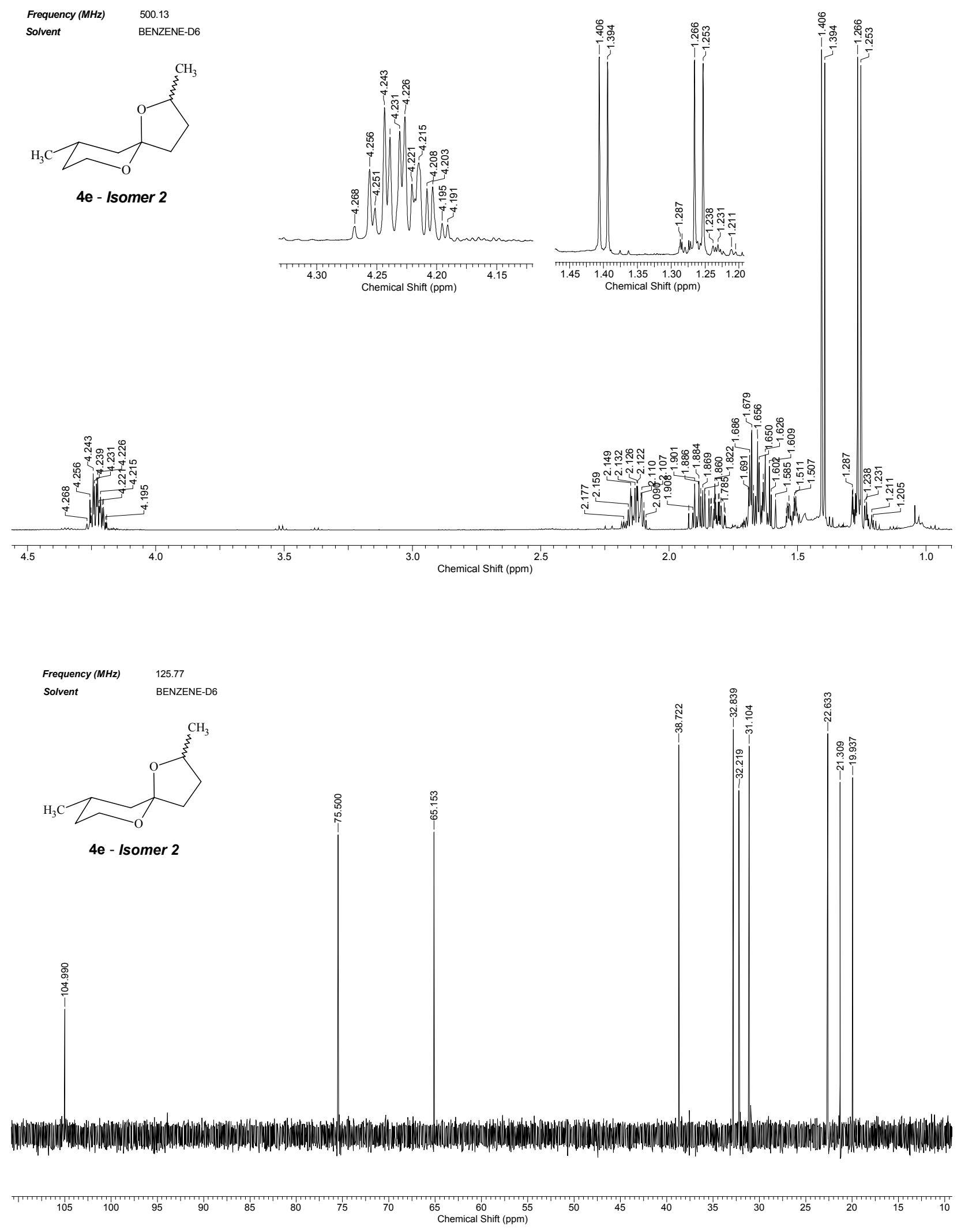

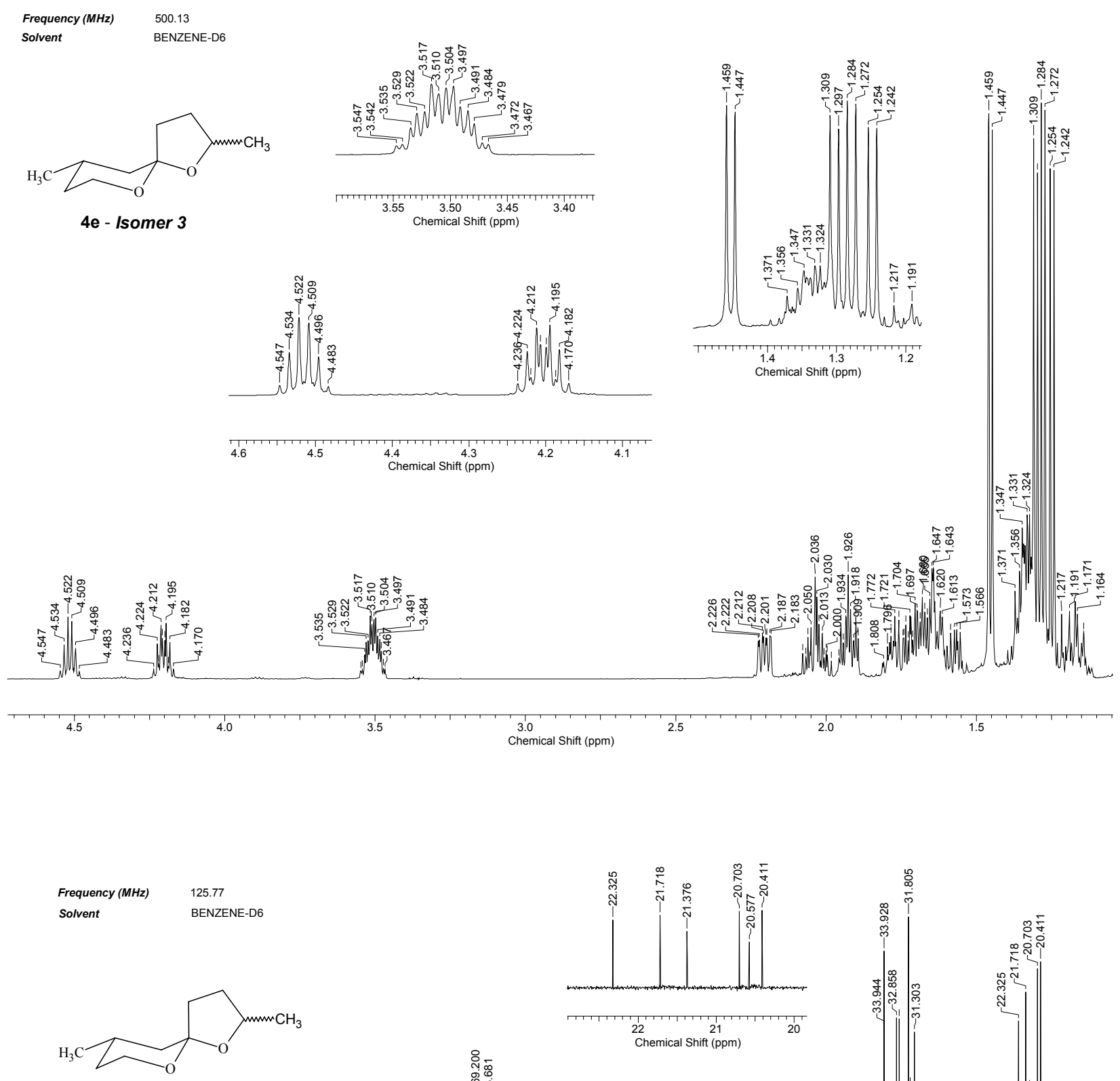

$4 \mathrm{e}-$ Isomer 3

ํ.
os
0.0
100
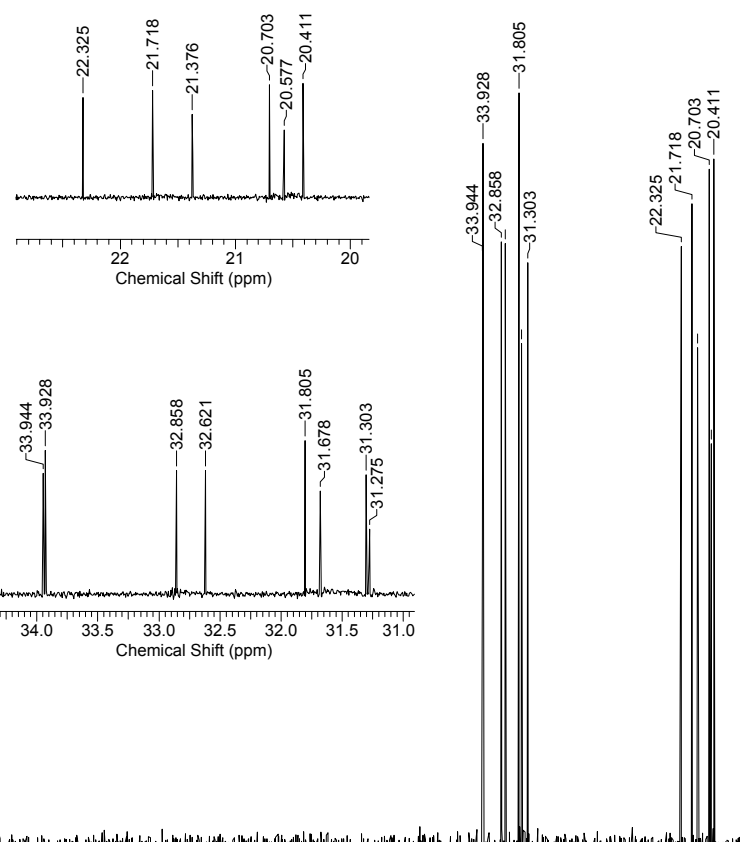

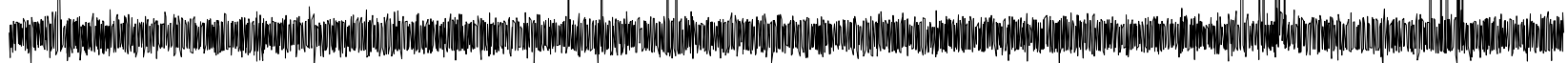
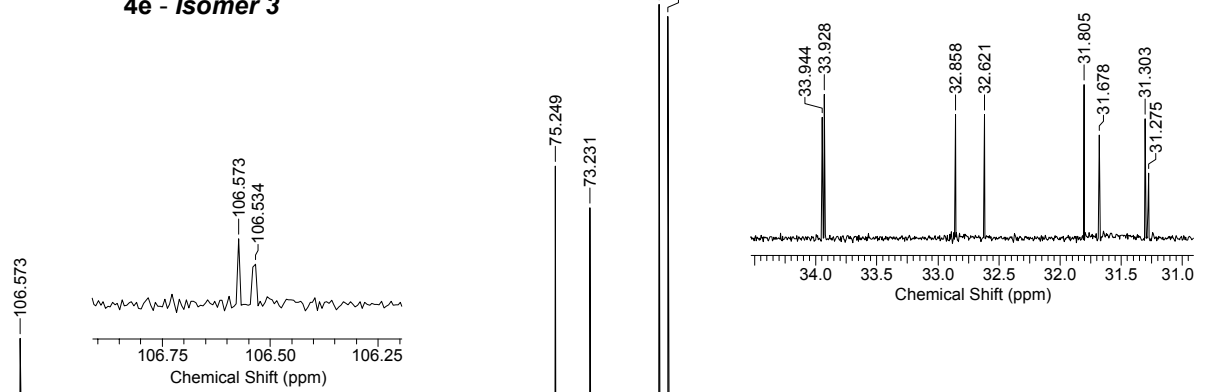

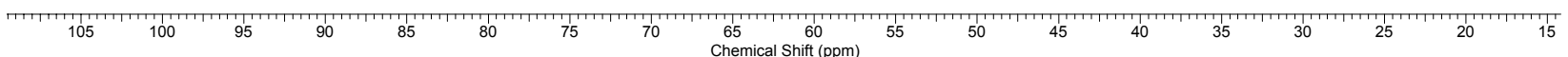




\section{Example 3:}

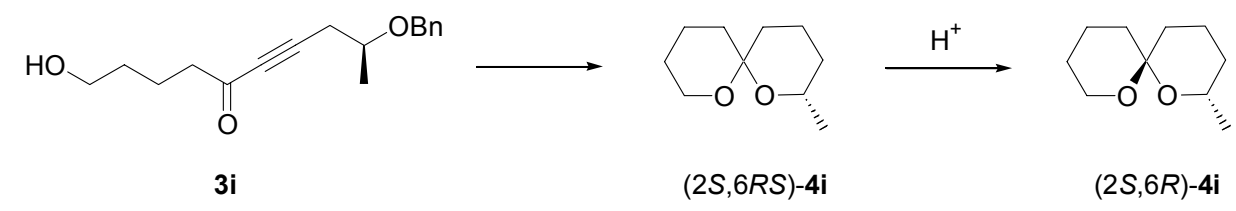

$\underline{\text { GC analyses: }}$

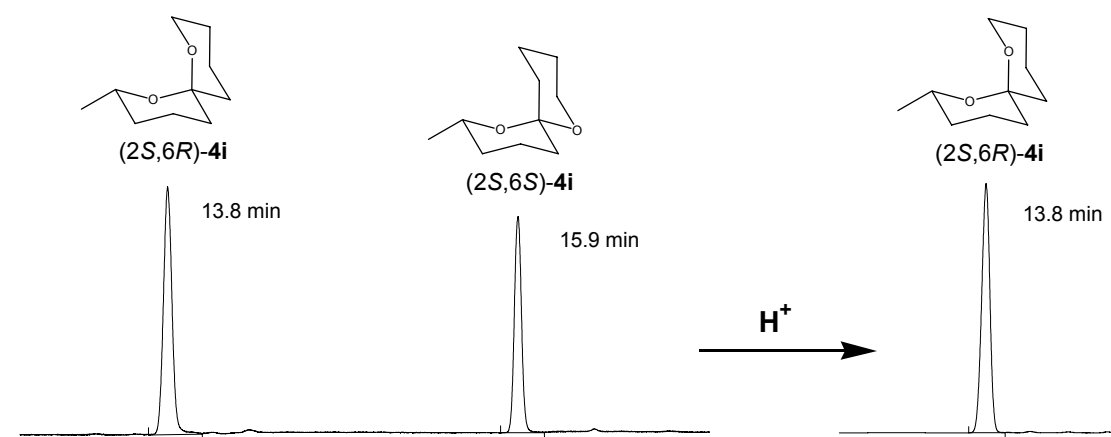

GC-MS:

MS-spectra were closely similar for both isomers.

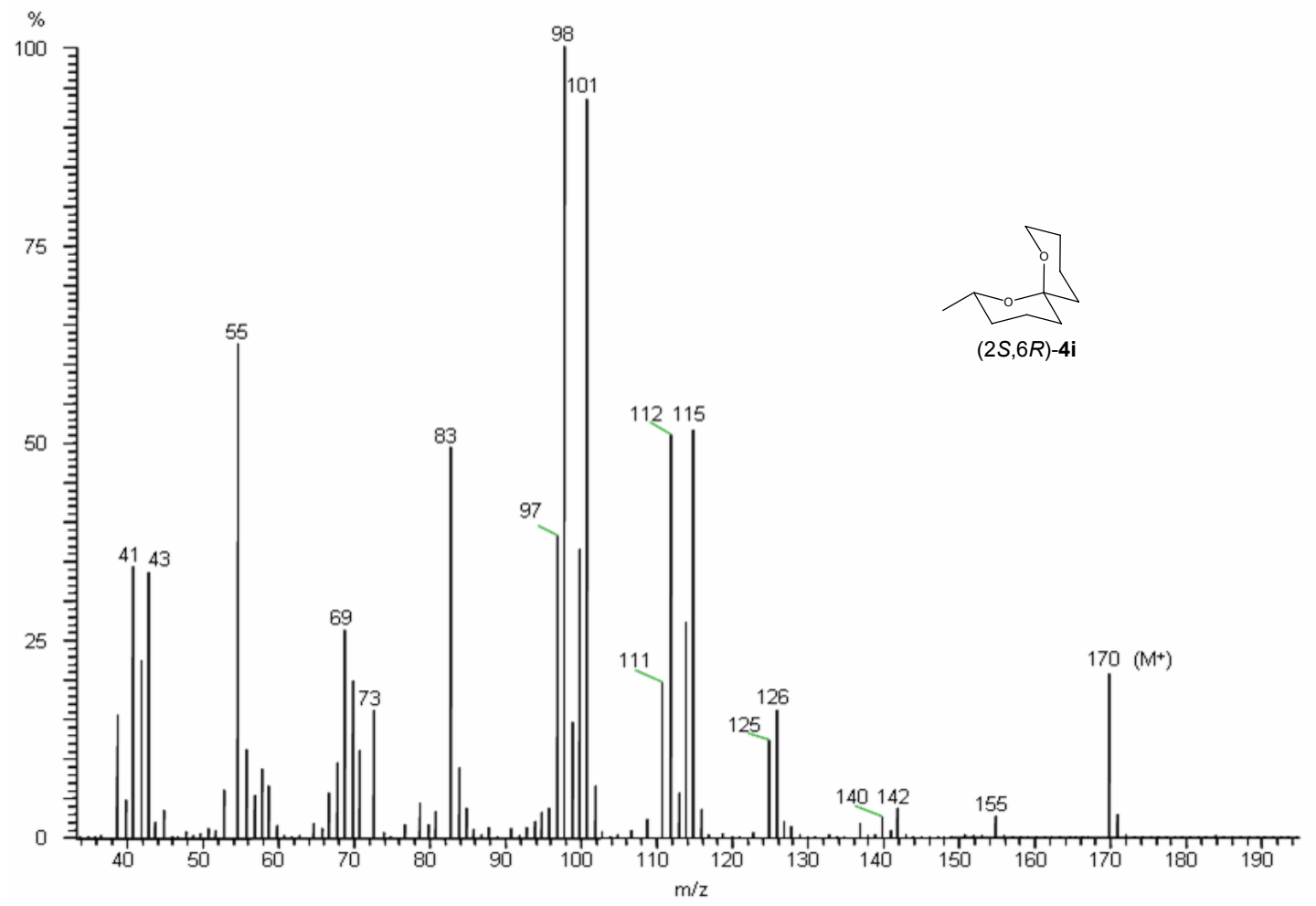


GC-IR:
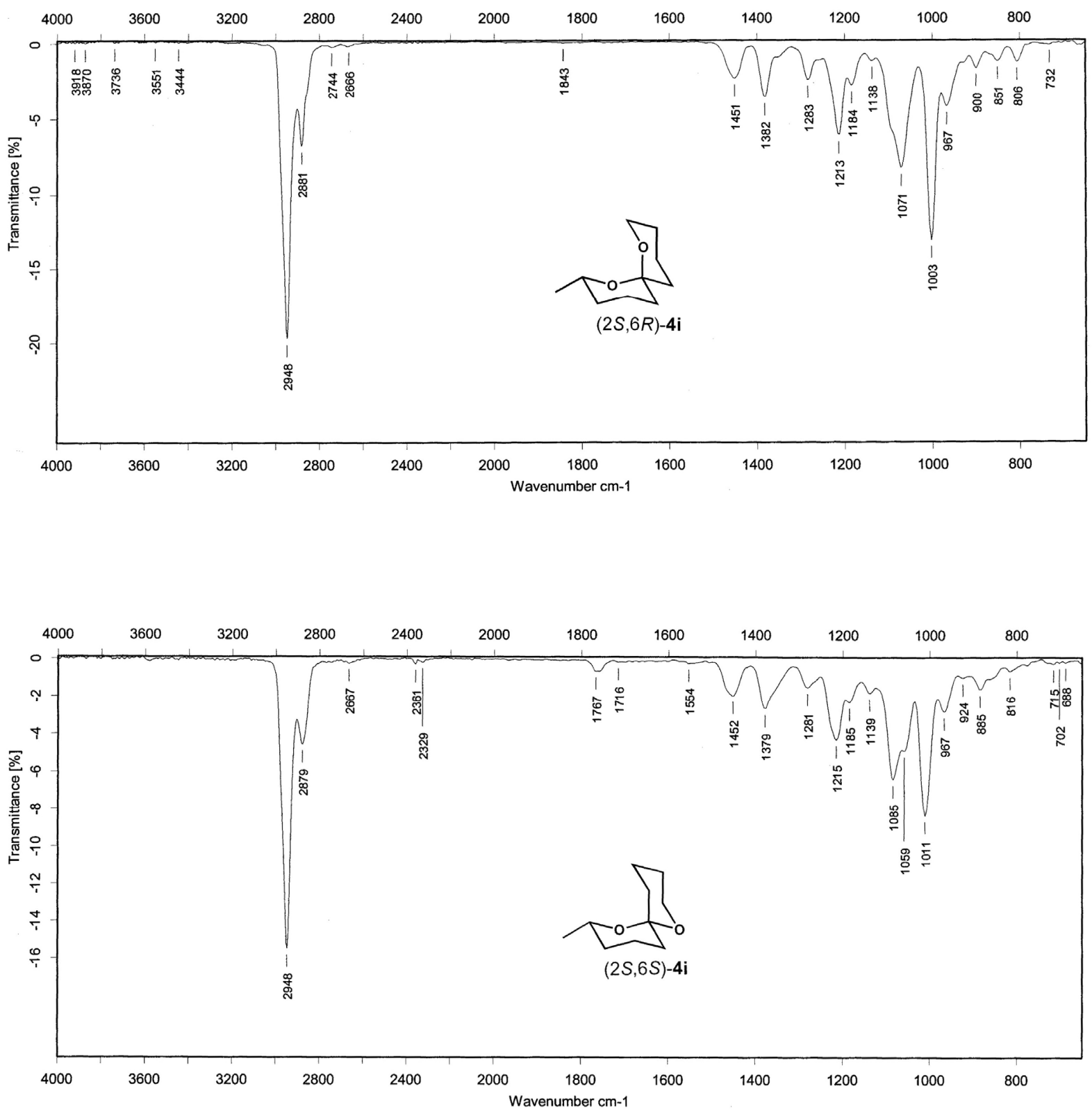


\section{$\underline{{ }^{1} \mathrm{H} \text { and }{ }^{13} \mathrm{C}-\mathrm{NMR} \text { spectra: }}$}
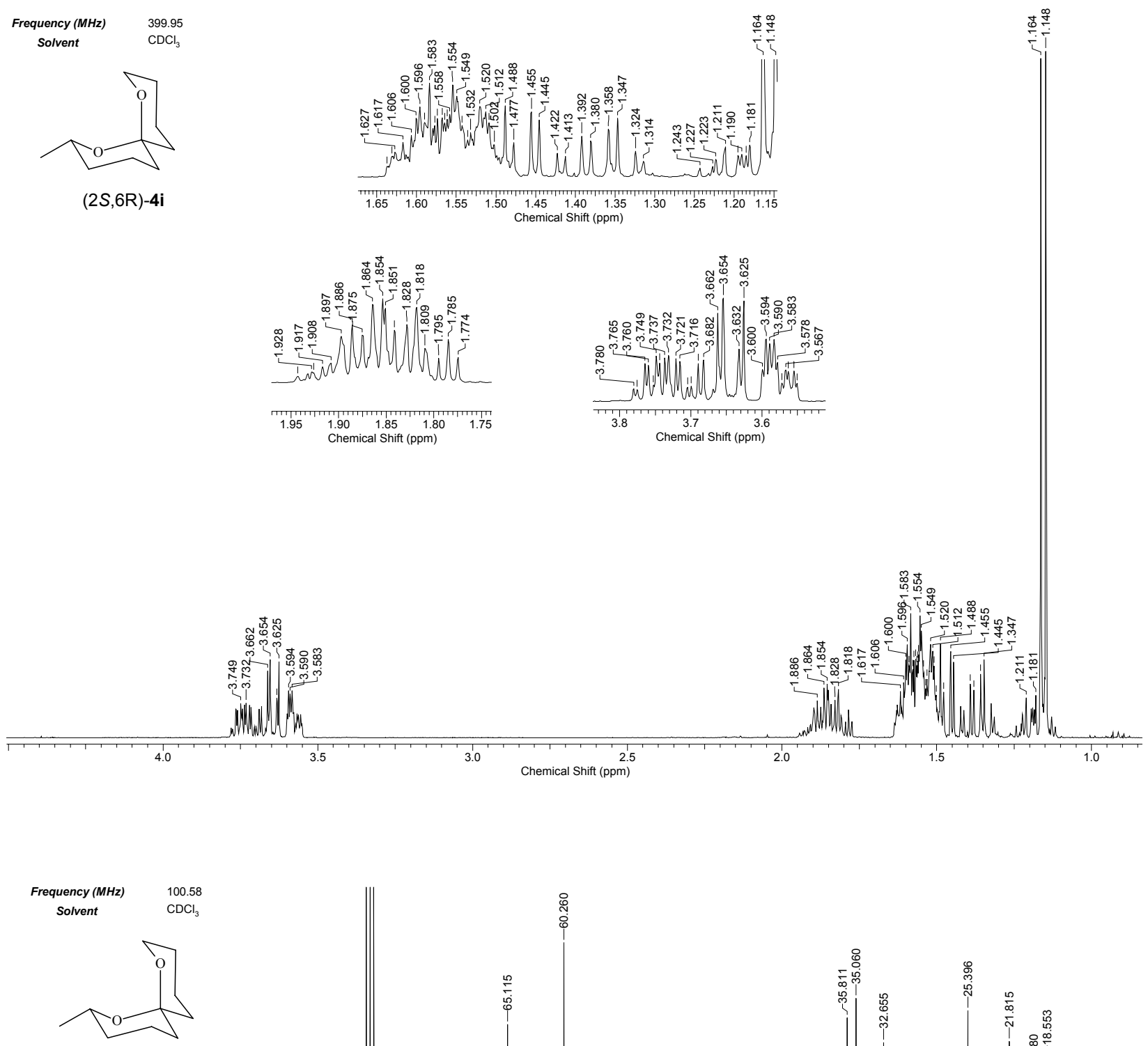

$(2 S, 6 R)-4 \mathbf{i}$

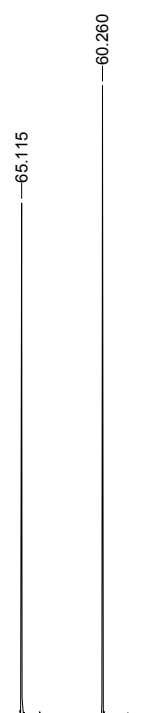

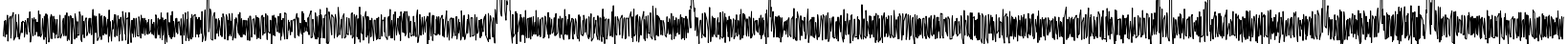

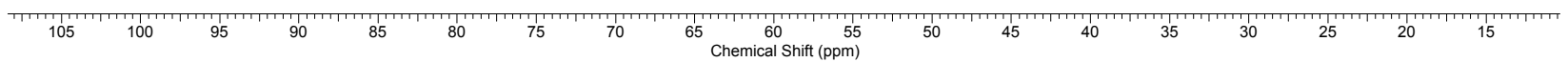


S18

Example 4:

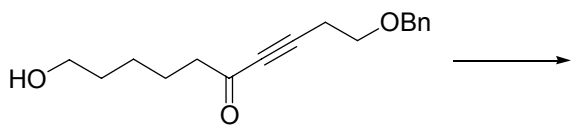

3j

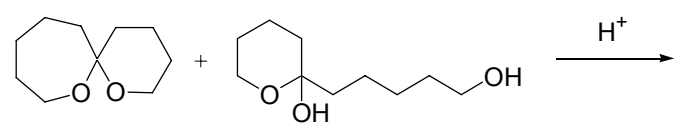

4j

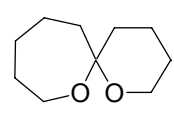

4j

GC analyses:

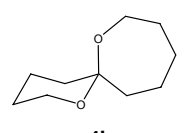

4j

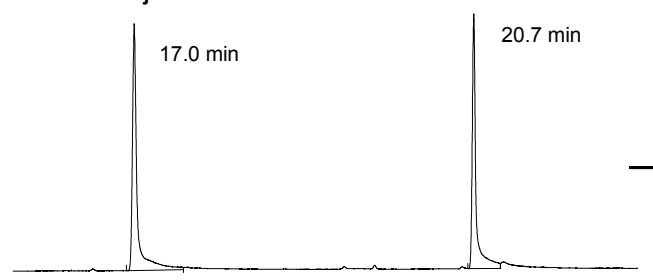

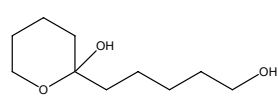

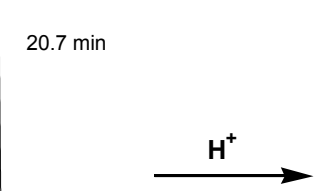

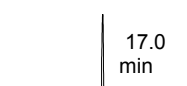

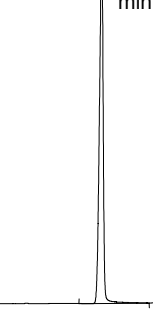

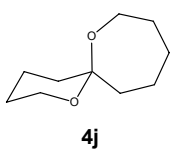

$20.7 \mathrm{~min}$

GC-MS:

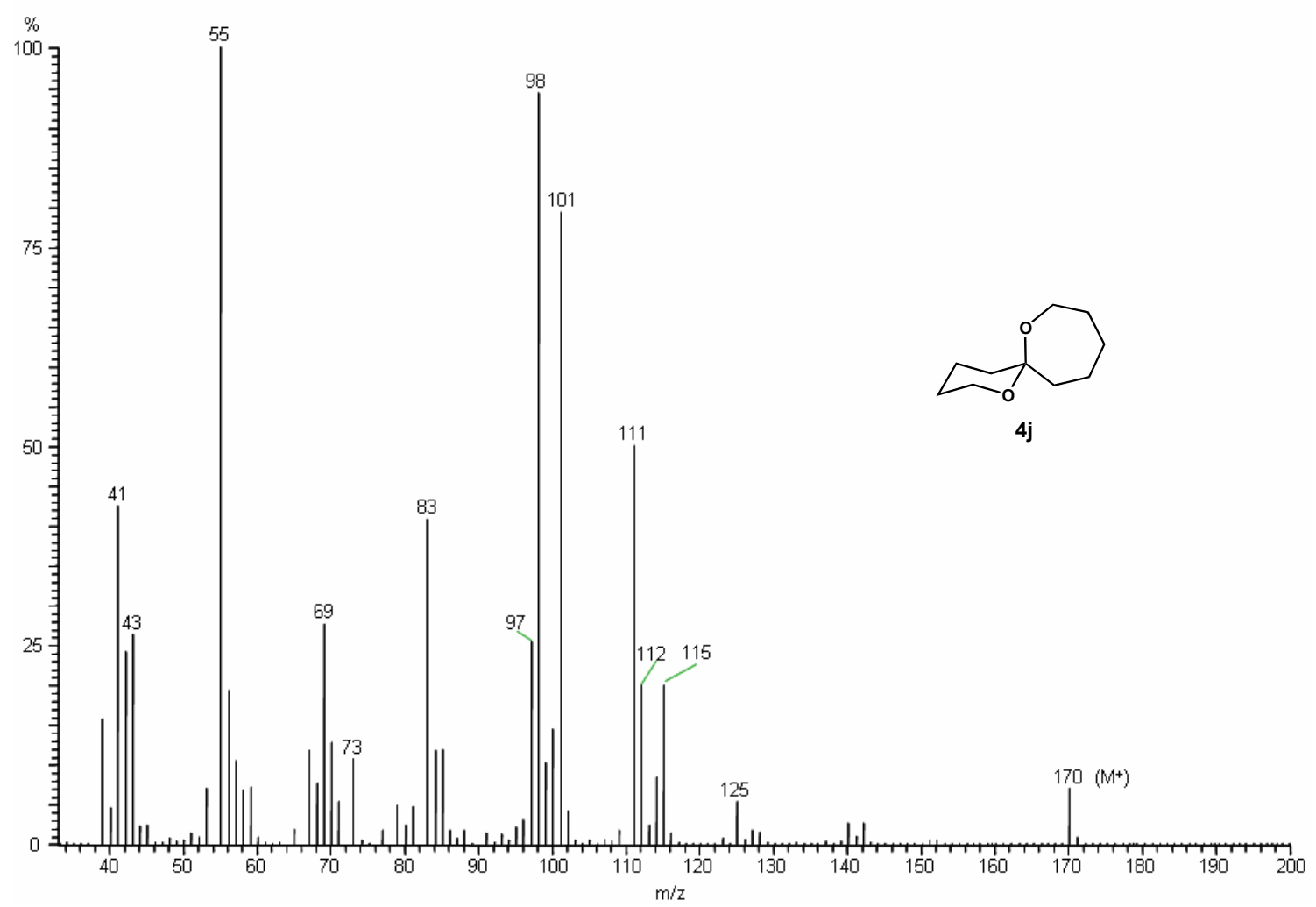


GC-IR:

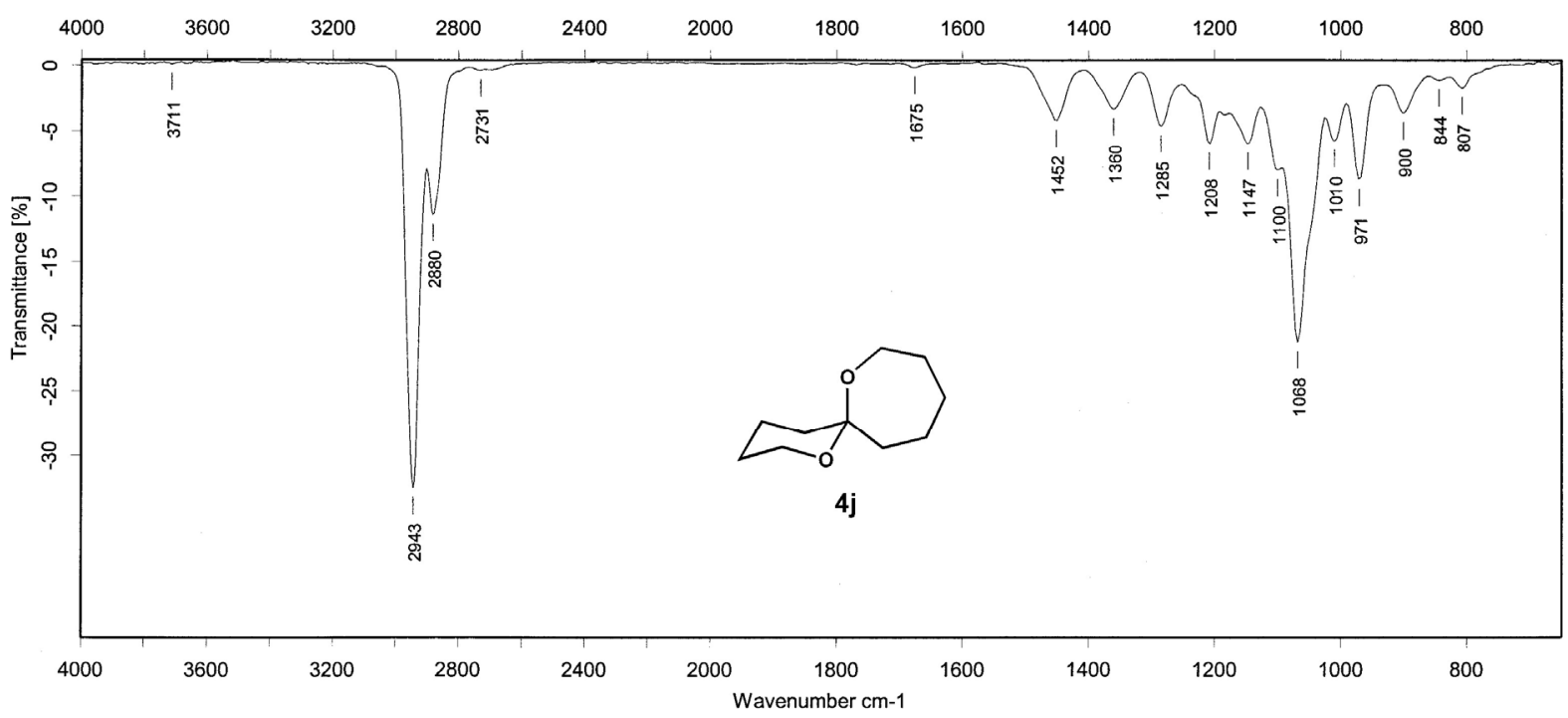

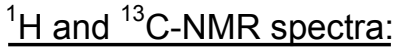

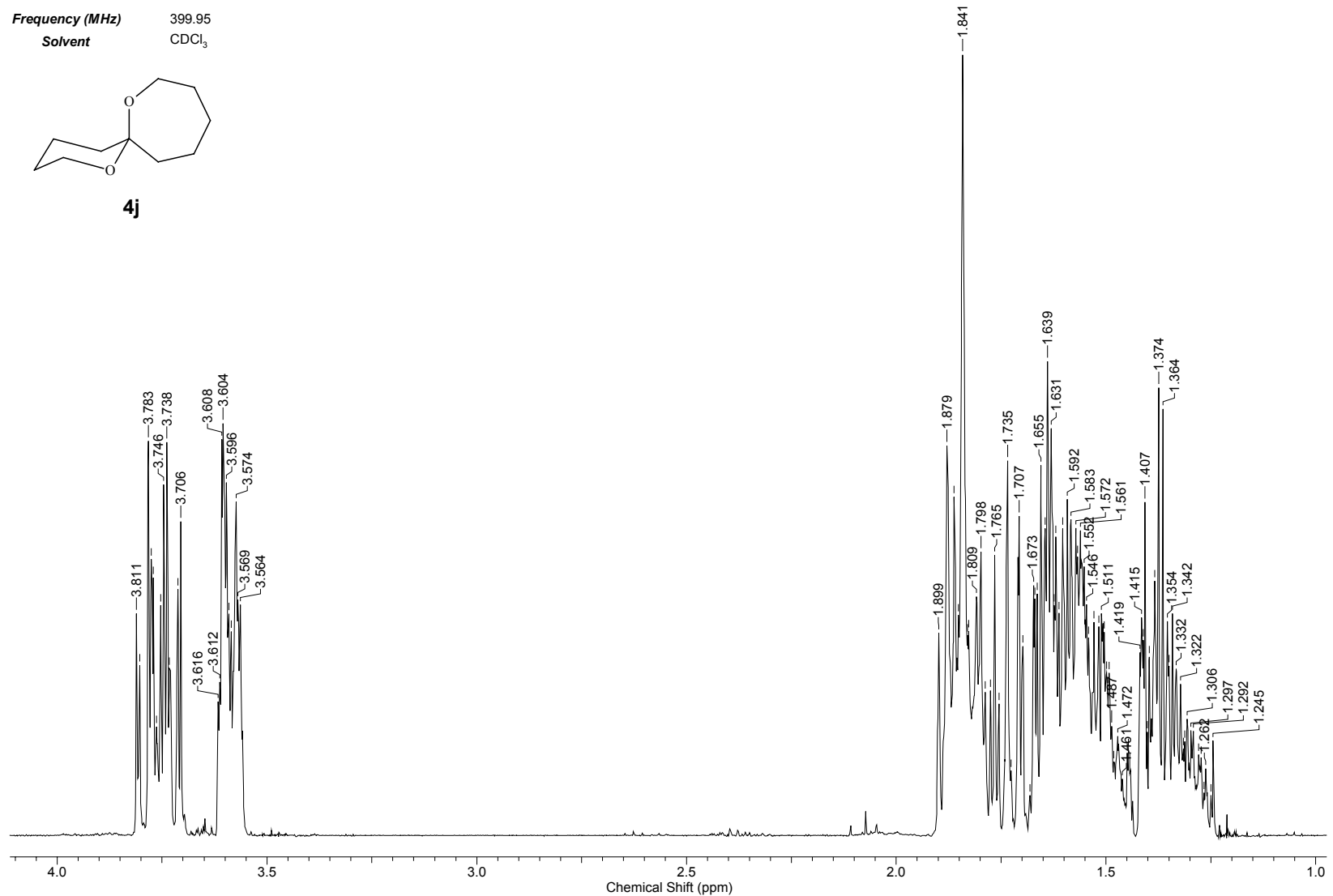



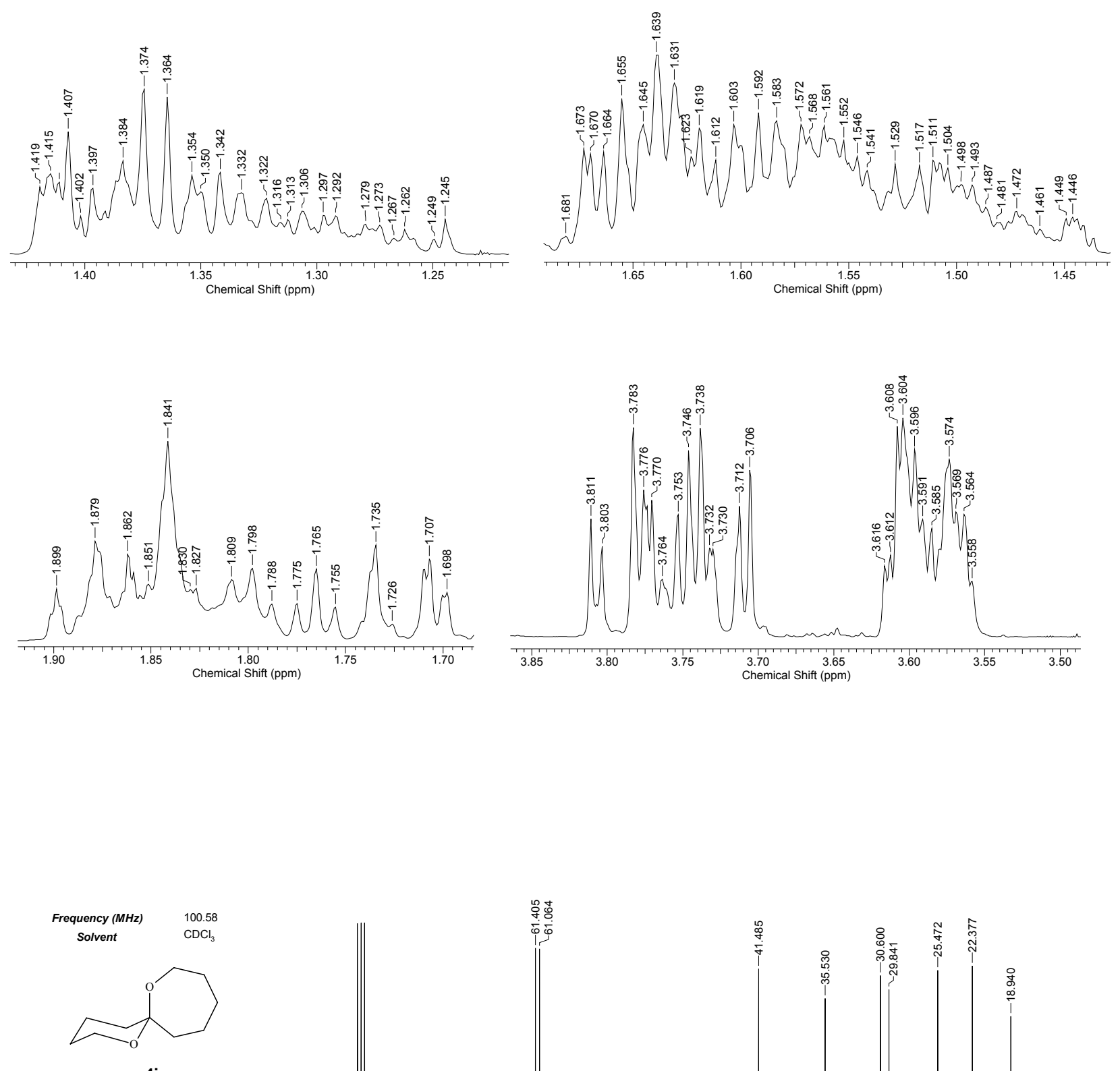

4j

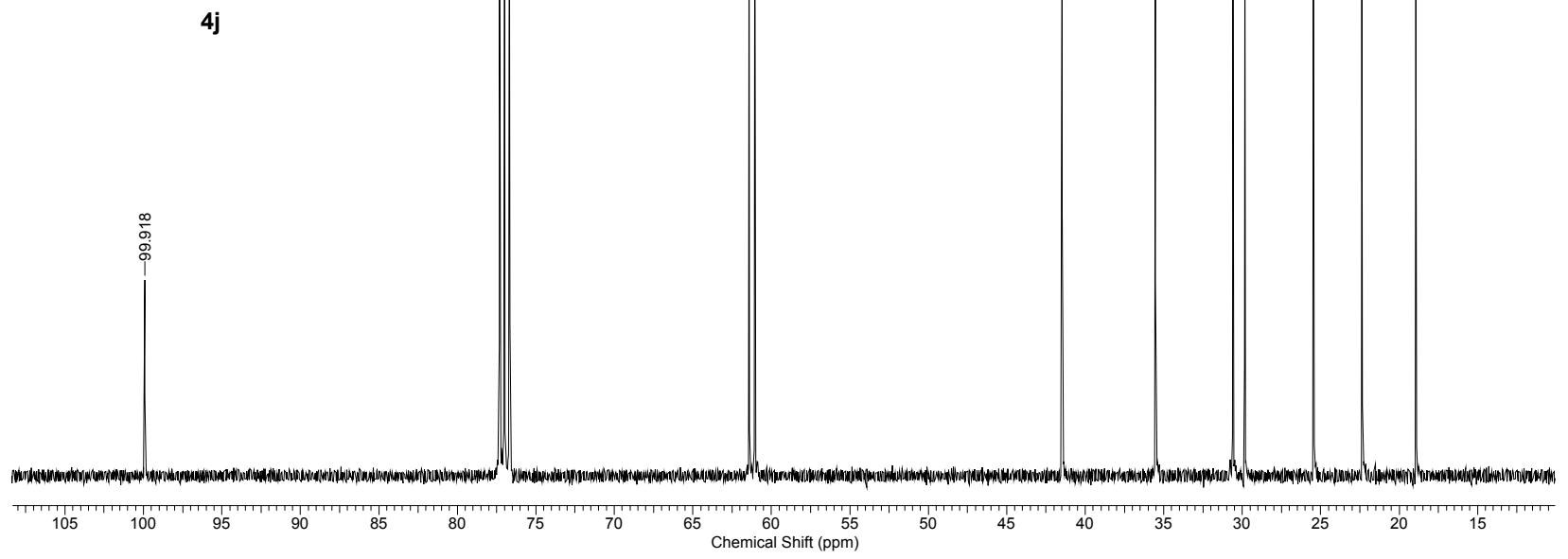




\section{Example 5:}

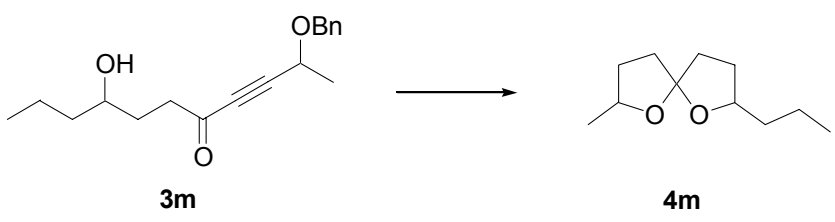

\section{GC analyses:}

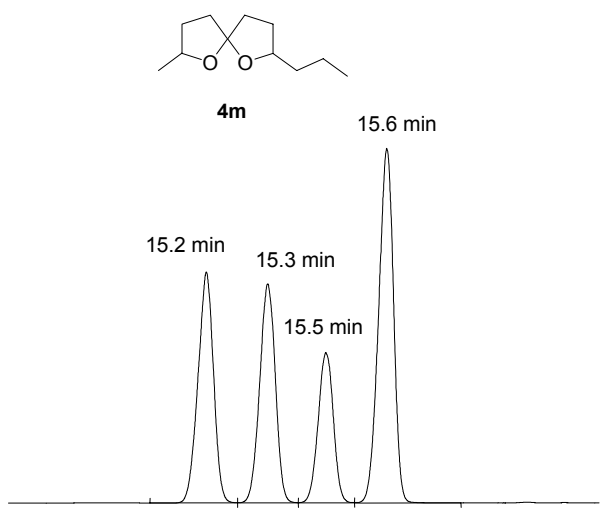

\section{GC-MS:}

MS-spectra were closely similar for all isomers.

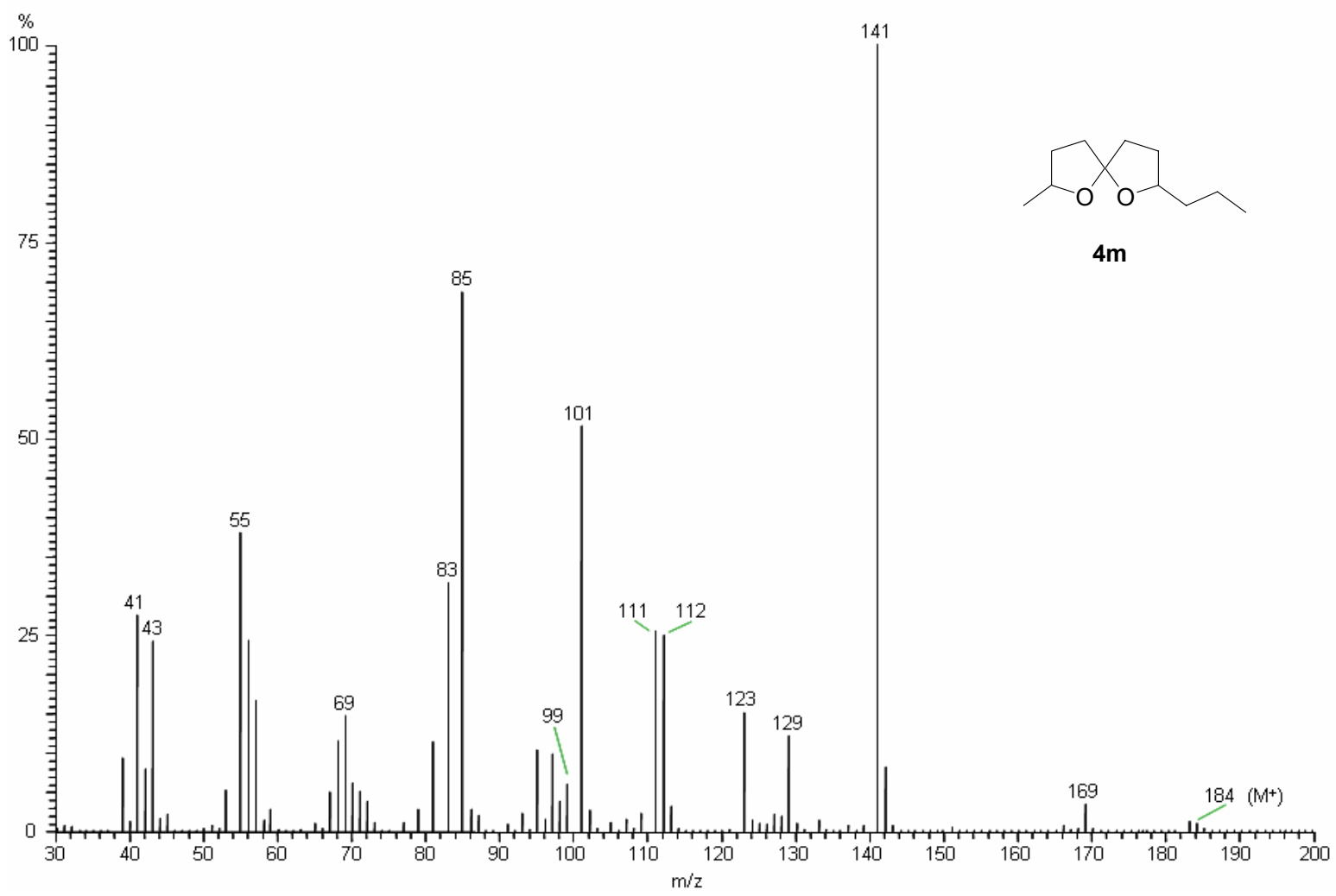


GC-IR:

The numbering of isomers follows the retention times order.
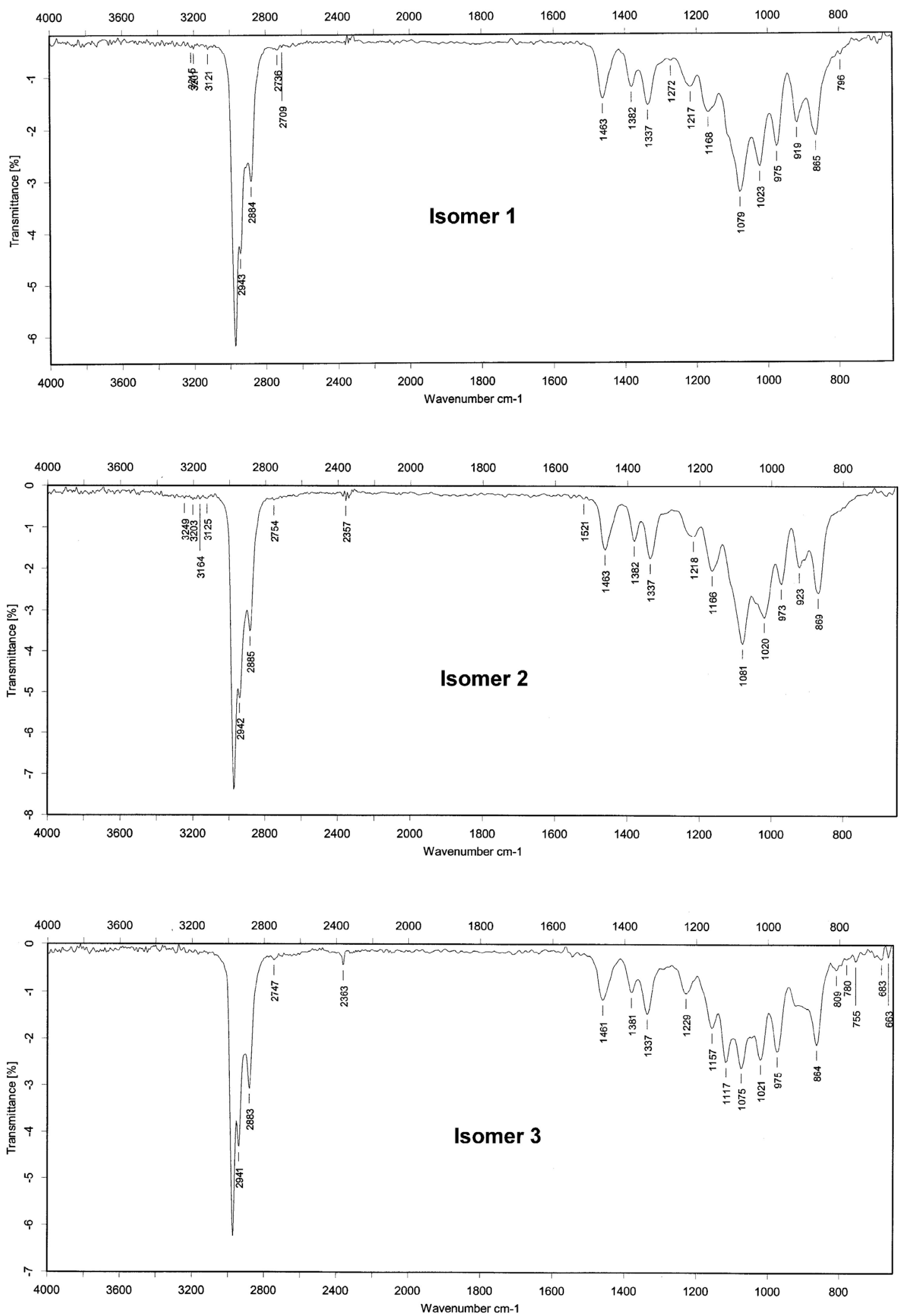
S23

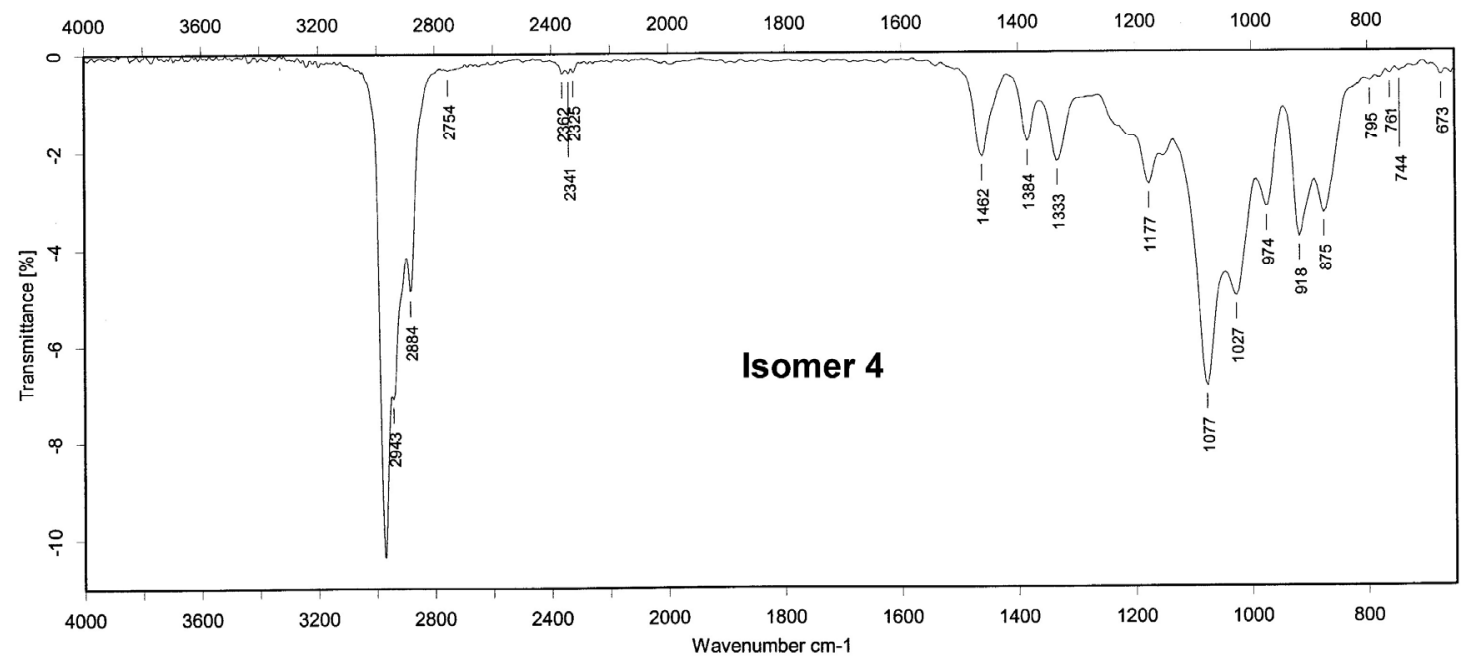

$\underline{ }{ }^{1} \mathrm{H}$ and ${ }^{13} \mathrm{C}-\mathrm{NMR}$ spectra:

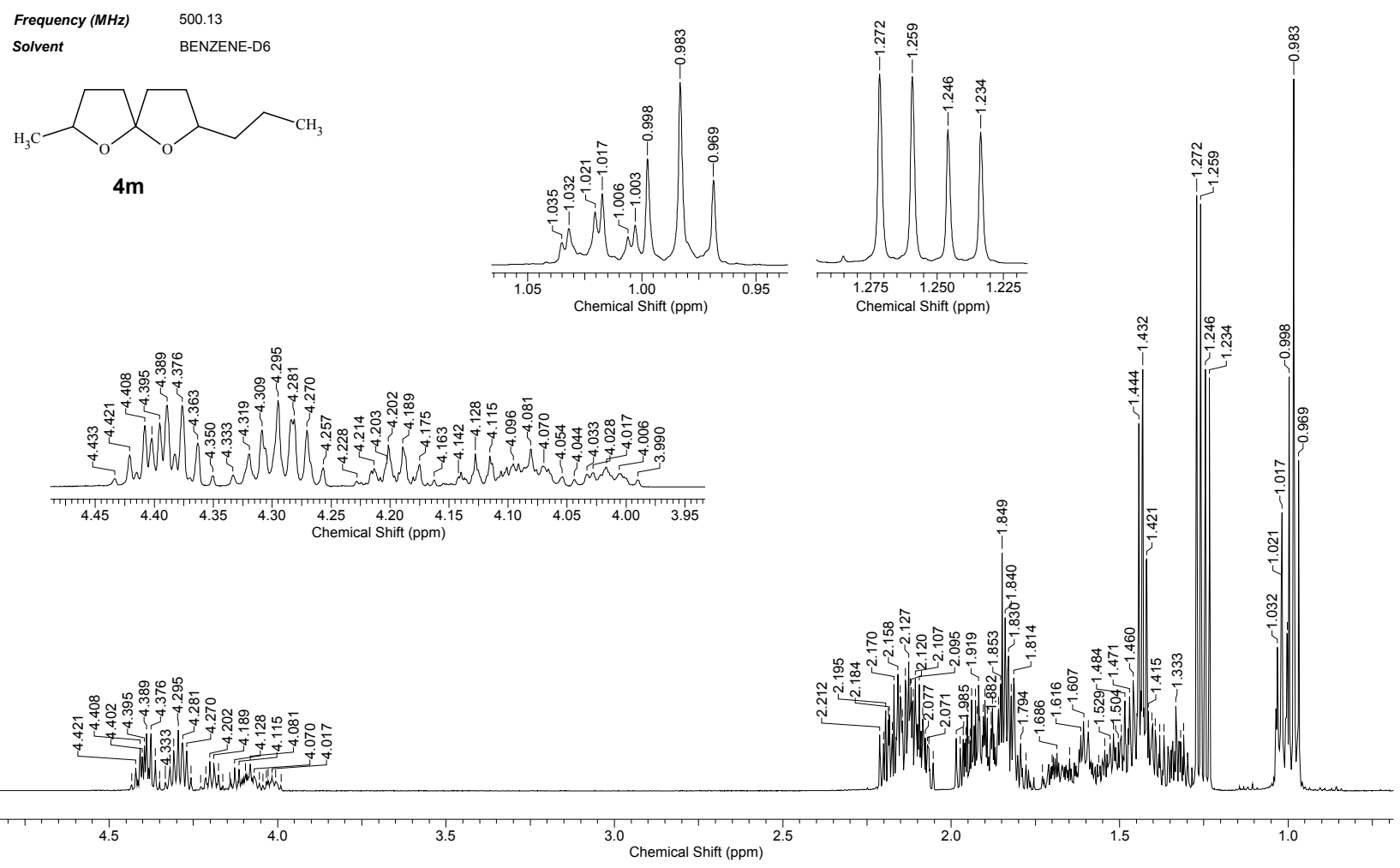




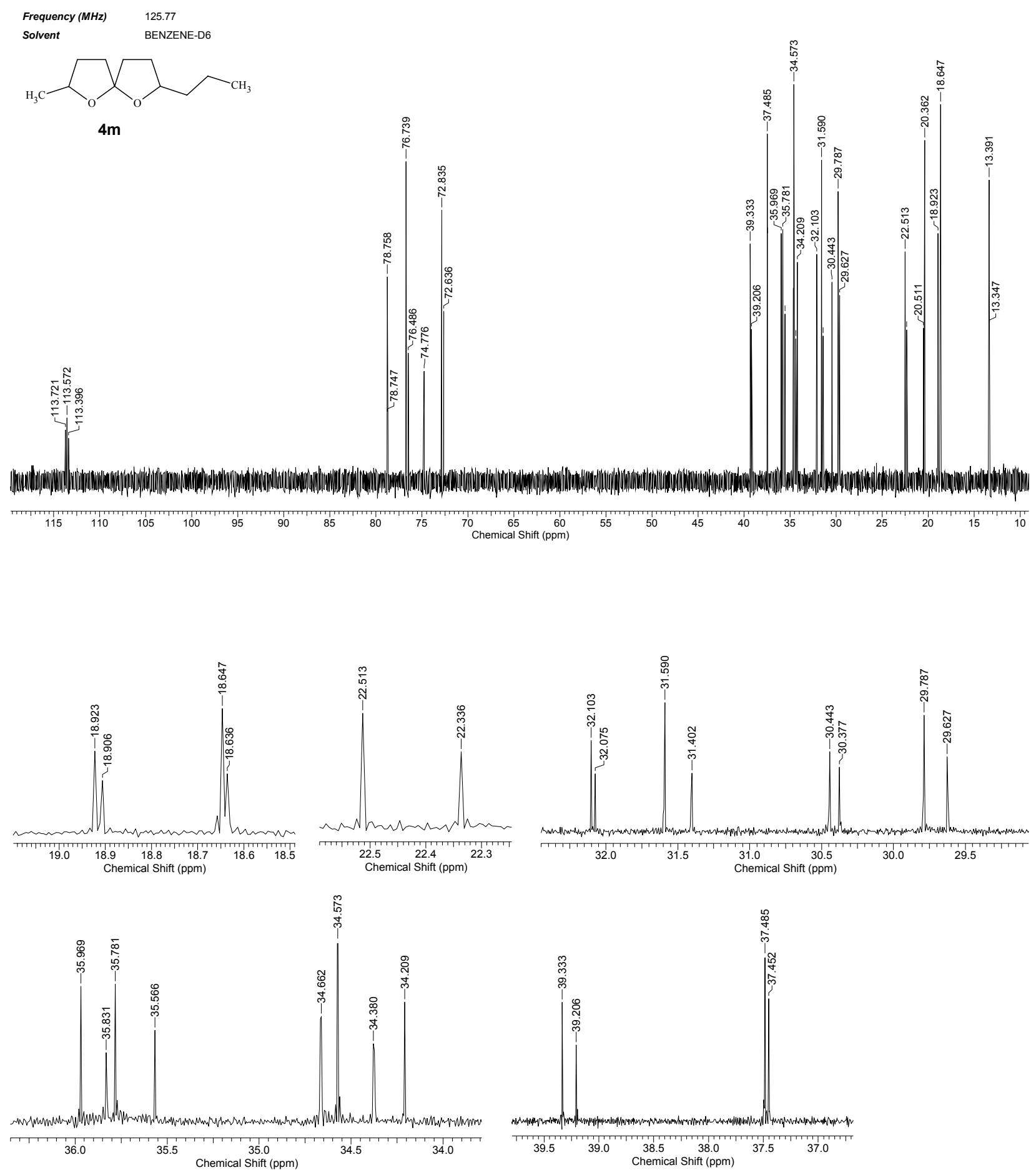

